\title{
Thermo-elastic aspects of dynamic nucleation
}

\author{
S.-C. Ngan ${ }^{(1)}$ and L. Truskinovsky ${ }^{(2)}$ \\ (1) Center for Magnetic Resonance Research, University of Minnesota, Minneapolis, MN55455, USA \\ e-mail: ngan@cmrr.umn.edu \\ ${ }^{(2)}$ Department of Aerospace Engineering and Mechanics, University of Minnesota, Minneapolis, MN55455, USA. \\ phone: 612 6240529, fax: 612 6261558, e-mail: trusk@aem.umn.edu
}

Keywords: dynamics (A), phase transformation (A), thermomechanical processes (A), stress waves (B), nucleation and growth (B)

\begin{abstract}
In spite of recent progress in our understanding of the absolute stability of elastic phases under loads, the generic presence of metastable configurations and the possibility of their dynamic breakdown remains a major problem in the mechanical theory of phase transitions in solids. In this paper, by considering the simplest one-dimensional model, we study the interplay between inertial and thermal effects associated with nucleation of a new phase, and address the crucial question concerning the size of a perturbation breaking metastability. We begin by reformulating the nucleation problem as a degenerate Riemann problem. By choosing a specific kinetic relation, originating from thermo-visco-capillary (TVC) regularization, we solve a self-similar problem analytically and demonstrate the existence of two types of solutions: with nucleation and without it. We then show that in the presence of a nonzero latent heat, solution with nucleation may by itself be non-unique. To understand the domain of attraction of different self-similar solutions with and without nucleation, we regularize the model and study numerically the full scale initial value problem with locally perturbed data. Through numerical experiments we present evidence that the TVC regularization is successful in removing deficiencies of the classical thermo-elastic model and is sufficient in specifying the limits of metastability.
\end{abstract}

\section{Introduction}

Recent interest in the dynamical response of multiphase solids has been stimulated by the broadening use of materials exhibiting "smart" or "active" behavior in various high frequency devices. As it is well known, the enhanced mechanical properties of these materials are due to martensitic phase transitions (see Otsuka and Wayman (1998) for a recent review). Although there has been considerable progress in recent

years in the understanding of the equilibrium or quasi-static properties of transforming solids, the dynamical picture remains mostly unclear and the foremost open problems in the mathematical structure of the theory concern the mechanisms of rate sensitivity. It is then not surprising that both kinetics and dynamics of phase transformations have recently been the subjects of intense interest (see for instance Abeyaratne et al.(1996), Rosakis and Knowles (1997), Truskinovsky (1997), Shield et al. (1997), Lin and Pence (1998), Ngan and Truskinovsky (1999), Vainchtein (1999), Slepyan (2001)). Most of the 
unresolved questions in this area of research have their roots in the problems of metastability and nucleation.

In this paper we focus on the thermo-elasto-dynamical aspects of the nucleation phenomenon. Radiation of sound accompanying martensitic phase transitions together with a high mobility of phase boundaries unambiguously point towards fully dynamical treatment of the transformation process. On the other hand, the presence of nonzero heat effects and the pronounced influence of heat release on the size and the structure of the hysteresis loops suggest that the adequate treatment of the problem must be fully thermodynamical. In the realistic case when the transformation process is sufficiently fast and thermal boundary layers are sufficiently narrow, the processes in the bulk can be considered adiabatic. This will be our main assumption in the rest of the paper.

To emphasize the ideas we employ the most elementary one-dimensional model of an elastic bar with non-convex elastic energy (Ericksen, 1975). By using this rather simplified framework we study a general scenario of homogeneous nucleation leading to an explosive decomposition of a metastable state. The mathematical problem reduces to the analysis of a degenerate Riemann problem with identical data on both sides of the nucleation site; the dynamics of the initial stage of the nucleation process and the associated generation of shock waves can then be modeled by the corresponding selfsimilar solutions. When the initial state is metastable, this degenerate Riemann problem is ill posed, exhibiting severe non-uniqueness associated with the ambiguity in the continuum description of both nucleation of the new phase and its growth. A nonuniqueness of this type was first noticed in the isothermal context by James (1980).

The growth aspect of this non-uniqueness is now well understood and is known to be remedied by assigning to the moving phase boundaries an additional admissibility condition often called a kinetic relation. Phenomenological (or ad hoc) kinetic relations were long known to physicists (e.g. "normal growth" condition) and have started to appear in the solid mechanics literature since the mid 70's (e.g. Dafermos (1973), Truskinovsky (1982, 1987), Shearer (1983), Abeyaratne and Knowles (1991a), Gurtin (1993)). As an alternative to specifying kinetics of growth phenomenologically, one can directly regularize the model and obtain an admissibility condition from the study of the fine structure of a transformation front. Here again several alternative regularization schemes have been suggested, including different variants of visco-elasticity and gradient elasticity, various phase field extensions and an assortment of discretizations (e.g. (Harten at al.(1976), Slepyan and Troiankina (1984), Slemrod and Flaherty (1986), Truskinovsky (1987, 1993b), Mihailescu and Suliciu (1992), Vainchtein and Rosakis (1999), Slepyan $(2000,2001))$. One of the most widely used approaches of this kind is the visco-capillary (VC) or thermo-visco-capillary (TVC) model introduced by Truskinovsky $(1982,1985)$ and Slemrod $(1983,1984 a)$ and further studied by Shearer (1986), Abeyaratne and Knowles (1991b), Truskinovsky (1993a,b, 1994,1997), Shearer and Yang (1995), Cockburn and Gao (1996), Rybka and Hoffmann (1998), Ngan and Truskinovsky (1999), LeFloch and Rohde (2000), Vainchtein (2001), and Chalons and LeFloch (2001) among others.

The nucleation aspect of the non-uniqueness, arising in the non-regularized degenerate Riemann problem, manifests itself through the presence of two types of solutions: a non-trivial one, describing nucleation and growth, and the trivial one, describing a system remaining in the metastable phase (e.g. James (1980), Shearer 
(1986), Truskinovsky $(1994,1997))$. To remedy this non-uniqueness one can again follow two paths: phenomenology or regularization. Along the phenomenological path, Abeyaratne and Knowles (1991) suggested that the choice between the trivial and the non-trivial solutions should be based on a postulate, which formally divides the metastable region into two parts: one, where the trivial solution is preferred, and another, where the dynamic continuation must be chosen. Combined with an appropriate phenomenological kinetic relation, this nucleation criterion was shown to guarantee uniqueness of solutions for a generic Riemann problem in a tri-linear thermo-elastic material.

In the present paper we argue that the non-uniqueness associated with nucleation can also be resolved through the regularization leading to more detailed description of the process at the micro-level. From the perspective of the regularized model one can reason that the two solutions of the degenerate Riemann problem, trivial and non-trivial, actually correspond to different initial data, even though at the level of resolution of the nonregularized problem, the initial data seem to be identical ${ }^{1}$. To illustrate this idea, we use the TVC regularization and augment the system of equations of adiabatic thermoelasticity by adding thermal conductivity, viscosity and gradient elasticity (weak nonlocality). In this regularized framework we demonstrate numerically that a localized perturbation of the original metastable state can generate two distinct dynamic regimes: one describing explosive nucleation and the other one exhibiting the decay of the perturbation. Contrary to the self-similar case, in the regularized setting the two regimes correspond to slightly different initial data. The analysis of the continuous dependence of these solutions on the initial data allows one to relate the nucleation phenomenon to the size of the domain of attraction of the regime producing new phase. As we show, a direct comparison of the classical and regularized approaches leads naturally to the nucleation criterion which is compatible with the kinetic relation in the sense that both originate from the same micro-mechanical model. Similar analysis of the nucleation in the discrete setting (lattice model) can be found in Balk et al. (2001a,b); the issue of direct nucleation of phase mixtures have been recently addressed in Ren and Truskinovsky (2000).

The outline of the paper is as follows. In Section 2, we provide a brief summary of results concerning governing equations and jump conditions in the non-regularized adiabatic problem. The failure of this theory to produce a unique solution of the nucleation problem is then established and the physical phenomena leading to the nonuniqueness are analyzed. To fix the deficiency of the classical approach, in Section 3 we regularize the problem and formulate the new system of equations containing higher derivatives of the main variables. Furthermore, to facilitate numerical studies in the latter parts of the paper, we specify a particular material model with cubic stress strain relation and maximally simplified temperature dependence. In Section 4, we discuss the traveling wave solutions of the regularized system and identify the associated kinetic relation. This kinetic relation is then used in Section 5, which contains a detailed analysis of the selfsimilar nucleation in the non-regularized problem. In Section 6 we formulate a numerical scheme which is then used to simulate dynamics in the regularized problem. A series of numerical experiments aimed at finding a critical perturbation is first discussed in the context of a simpler isothermal problem. We then simulate full scale adiabatic nucleation

\footnotetext{
${ }^{1}$ Observations in Truskinovsky (1994) that sufficiently fast moving phase boundaries are unstable with respect to highly localized finite perturbations illustrate the same idea.
} 
and discover that only one of the variety of self-similar solutions discussed in Section 5 plays a role of an attractor in the regularized initial value problem with locally perturbed data. Our main conclusions are summarized in the final section of the paper.

\section{Preliminaries}

In this section we recall some results concerning elasto-dynamics of phase transitions in one dimension and reformulate the nucleation problem as a degenerate Riemann problem. We refer the reader to Truskinovsky (1993a, b, 1994, 1997), Nhan and Truskinovsky (1999) and the references cited therein for additional background and details.

\subsection{Equations and jump conditions}

Consider a time-dependent longitudinal deformation of a homogeneous thermoelastic bar with a unit cross section. Assume for simplicity that the referential density is equal to unity, and let $u(x, t)$ be displacement of a reference point $x$ at time $t$. Our main variables will be the strain $w=\partial u / \partial x$ and the particle velocity $v=\partial u / \partial t$.

Adiabatic model. Suppose that heat conductivity can be neglected outside the narrow transition zones. Then, the standard balances of mass, linear momentum and energy yield

$$
\frac{\partial w}{\partial t}=\frac{\partial v}{\partial x}, \frac{\partial v}{\partial t}=\frac{\partial \sigma}{\partial x}, \frac{\partial e}{\partial t}=\sigma \frac{\partial v}{\partial x}
$$

where $e(w, s)$ is specific internal energy, $s$ is entropy, and $\sigma=\partial e / \partial w$ is stress. On the shocks and phase discontinuities, the system (2.1.1) must be supplemented by the Rankine-Hugoniot jump conditions

$$
D[w]+[v]=0, D[v]+[\sigma]=0, D\left[e+1 / 2 v^{2}\right]+[\sigma]=0
$$

Here, $D$ is the Lagrangian velocity of the discontinuity; for the jump, we use a standard notation: [ ] $=()_{+}-()_{-}$, where "+" corresponds to the state ahead of the discontinuity. A convenient form of (2.1.2) can be obtained if the particle velocity $v$ is eliminated. Then, for $D \neq 0$, we get

$$
[\sigma]-D^{2}[w]=0,[e]-\{\sigma\}[w]=0
$$

where \{\}$=1 / 2\left(()_{+}+()_{-}\right)$denotes the average of the two limiting values. The entropy inequality can now be written as

$$
\Re:=D G \geq 0
$$


where

$$
G=[f]-\{\sigma\}[w]+[T]\{s\},
$$

is the configurational force conjugate to the velocity of the discontinuity, $T=\partial e / \partial s$ is temperature, and $f=e-T s$ is specific free energy; an alternative expression

$$
G=\{T\}[s]
$$

emphasizes the entropic nature of this parameter.

Isothermal model. Parallel to the adiabatic model, a simpler isothermal model with $T \equiv T_{0}=$ const will be considered as prototypical. In the isothermal case the main system of equations takes the form

$$
\frac{\partial w}{\partial t}=\frac{\partial v}{\partial x}, \frac{\partial v}{\partial t}=\frac{\partial \sigma}{\partial x}
$$

where now $\sigma=\partial f(w, T) / \partial w$. The Rankine-Hugoniot jump conditions for the isothermal case read

$$
D[w]+[v]=0, D[v]+[\sigma]=0 .
$$

The entropy inequality takes the form

$$
\Re=D G \geq 0
$$

where the expression for the configurational force

$$
G=[f]-\{\sigma\}[w]
$$

follows from (2.1.5) under the assumption that $[T]=0$.

\subsection{Constitutive assumptions}

Our main interest concerns materials that can support two phases. We begin with the isothermal constitutive model and following the original idea of Ericksen (1975), assume that the free energy at a constant temperature $f\left(w, T_{0}\right)$ is a non-convex function of $w$. In particular, suppose that $f^{\prime \prime}(w)>0$ for $w<\alpha$ (phase 1) and $w>\beta$ (phase 2) and $f^{\prime \prime}(w)<0$ for $\alpha<w<\beta$ (spinodal region). The corresponding stress-strain relation $\sigma=f^{\prime}\left(w, T_{0}\right)$ is non-monotone (see Fig. 1), and one can formally define the equilibrium (Maxwell) stress $\sigma_{M}$ and the equilibrium strains $a_{t}$ and $b_{t}$ in such a way that

$$
\begin{gathered}
\sigma\left(a_{t}, T_{a}\right)=\sigma\left(b_{t}, T_{b}\right)=\sigma_{M} \\
f\left(a_{t}, T_{a}\right)-f\left(b_{t}, T_{b}\right)=\sigma\left(a_{t}, T_{a}\right)\left(a_{t}-b_{t}\right)
\end{gathered}
$$




$$
T_{a}=T_{b}=T_{0}
$$

By definition, the boundaries of the spinodal region $\alpha$ and $\beta$ mark the states with zero isothermal sound velocity

$$
c_{t}^{2}=\frac{\partial \sigma(w, T)}{\partial w} .
$$

The two regions $a_{t}<w<\alpha$ (in phase 1) and $\beta<w<b_{t}$ (in phase 2) are known as the domains of metastability (e.g. Ericksen (1975)). Finding a quantitative measure of the reserve of stability for the system in the metastable states constitutes the main subject of the present paper.

To extend the model to the adiabatic case, we must specify the non-isothermal part of the constitutive model. Consider first the Rankine-Hugoniot conditions (2.1.3) and suppose that the stress and strain $\left(\sigma_{+}, w_{+}\right)$in front of the discontinuity are prescribed. Then equations (2.1.3) describe two sets of points on the $(\sigma, w)$ plane: Rayleigh line, given by $\left(2.1 .3_{1}\right)$ and Hugoniot adiabat, given by $\left(2.1 .3_{2}\right)$. The two curves intersect at $\left(\sigma_{+}, w_{+}\right)$and possibly at one or several other points. To characterize the material, we assume that the Rayleigh line and the Hugoniot adiabat have up to three intersections, as it is schematically shown in Fig. 2. It is not hard to see that if specific heat at constant strain $C_{V}=\partial e(w, T) / \partial T$ is sufficiently large, this behavior is a direct consequence of the non-convexity of the free energy at constant temperature.

In the adiabatic context, conditions of phase equilibrium analogous to (2.2.1) can be written as

$$
\begin{gathered}
\sigma\left(a_{s}, T_{a}\right)=\sigma\left(b_{s}, T_{b}\right), \\
e\left(a_{s}, T_{a}\right)-e\left(b_{s}, T_{b}\right)=\sigma\left(a_{s}, T_{a}\right)\left(a_{s}-b_{s}\right), \\
s\left(b_{s}, T_{b}\right)=s\left(a_{s}, T_{a}\right) .
\end{gathered}
$$

Stability analysis of the homogeneous configurations suggests that the adiabatic analog of the spinodal region should contain configurations with

$$
c_{S}^{2} \equiv \frac{\partial \sigma(w, s)}{\partial w}<0 .
$$

The adiabatic metastability regions are then located between the adiabatic Maxwell states defined by (2.2.3) and the limits of the adiabatic spinodal region, specified by the condition $c_{S}=0$. 


\subsection{Degenerate Riemann problem}

Adiabatic case. Consider a bar of infinite extent in a homogeneous configuration with constant strain $w_{0}$, constant temperature $T_{0}$ and zero velocity $v_{0}=0$. This prescribes the following set of initial data for the system $(2.1 .1,2.1 .2,2.1 .4)$

$$
(w(x, 0), T(x, 0), v(x, 0))=\left(w_{0}, T_{0}, 0\right) .
$$

The initial value problem has a trivial solution

$$
w(x, t) \equiv w_{0}, T(x, t) \equiv T_{0}, v(x, t) \equiv 0 .
$$

Now, to model a nucleation event, we choose an arbitrary point $x=x_{0}$ and prescribe the same initial data everywhere except for this point. In other words,

$$
(w(x, 0), T(x, 0), v(x, 0))=\left\{\begin{array}{l}
\left(w_{0}, T_{0}, 0\right), \text { for } x>x_{0} \\
\left(w_{0}, T_{0}, 0\right), \text { for } x<x_{0}
\end{array},\right.
$$

which agrees with (2.3.1) everywhere outside $x=x_{0}$. We note that the configuration at point $x_{0}$ is left unspecified.

The initial value problem (2.3.3) belongs to a class of Riemann problems with piecewise constant initial data. Our particular Riemann problem is degenerate because the initial data on both sides of the discontinuity point are identical. If a non-trivial solution to this problem exists, it must be of the form

$$
w=w(\varsigma), T=T(\varsigma), v=v(\varsigma)
$$

where $\varsigma=\left(x-x_{0}\right) / t$. As it is well known, such a solution can be represented by a combination of the homogeneous states separated by jump discontinuities and/or centered Riemann waves.

Isothermal case. For the corresponding isothermal problem (2.1.7, 2.1.8, 2.1.9), the Riemann data, analogous to (2.3.3), take the form

$$
(w(x, 0), v(x, 0))=\left\{\begin{array}{l}
\left(w_{0}, 0\right), \text { for } x>x_{0} \\
\left(w_{0}, 0\right), \text { for } x<x_{0}
\end{array} .\right.
$$

The isothermal problem is simpler than the adiabatic one and we shall proceed by first constructing an explicit solution for this case. The analysis shows that if the initial state is in a metastable region within the phase 1, the Riemann problem (2.3.5) has a non-trivial self-similar solution, which corresponds to the nucleation and growth of the phase 2 


$$
w(x, t), v(x, t)=\left\{\begin{array}{cc}
w_{-}, 0 \quad, \quad\left|x-x_{0}\right|<D_{p} t \\
w_{+}, \pm v_{+} & D_{p} t<\left|x-x_{0}\right|<D_{s} t . \\
w_{0}, 0 \quad, & D_{s} t<\left|x-x_{0}\right|
\end{array}\right.
$$

These formulas describe the emission of two symmetric shock waves followed by the two symmetric subsonic phase boundaries. The situation is illustrated in Fig. 3.

To specify the solution, one has to solve for the five parameters $w_{+}, w_{-}, v_{+}, D_{s}$ and $D_{p}$. Since the constant states satisfy balance equations automatically, the only restrictions are provided by the four jump conditions (2.1.8). The entropy inequality (2.1.9) is necessarily satisfied for the shock wave precursors (moving with the speed $\pm D_{s}$ ) and is satisfied for the phase boundaries (moving with speeds $\pm D_{p}$ ) if the area $A_{1}$ in Fig. 3 is smaller than the area $A_{2}$. The only restriction imposed by the RankineHugoniot conditions is that the areas of the rectangle abcd and aefg in Fig. 3 are equal. One can see that the information contained in $(2.1 .7,2.1 .8$ and 2.1.9) is not sufficient to find the unknowns uniquely and instead, we obtain a one-parameter family of solutions. By taking into account the arbitrariness of $x_{0}$, the family of admissible solutions becomes two-parametric.

Adiabatic case. Now, we can return to the adiabatic problem (2.3.3). Given the structure of the Hugoniot adiabat, a non-trivial solution describing the explosive nucleation can be written in the form (see Fig. 4):

$$
w(x, t), T(x, t), v(x, t)=\left\{\begin{array}{cc}
w_{-}, T_{-}, 0 \quad, \quad\left|x-x_{0}\right|<D_{p} t \\
w_{+}, T_{+}, \pm v_{+} & D_{p} t<\left|x-x_{0}\right|<D_{s} t \\
w_{0}, T_{0}, 0 & , \quad D_{s} t<\left|x-x_{0}\right|
\end{array}\right.
$$

To specify this solution one has to solve for the seven parameters $w_{+}, w_{-}, v_{+}, T_{+}, T_{-}, D_{s}$ and $D_{p}$ and the only restrictions are those provided by the six jump conditions (2.1.2). Again, one can show that these constraints are not sufficient to find all the unknowns which leaves us with a two-parameter family of solutions.

\subsection{Growth}

Assume first that the point $x_{0}$ is given. Then in order to determine the constants $w_{+}, w_{-}, v_{+}, T_{+}, T_{-}, D_{s}$ and $D_{p}$ in the adiabatic problem one needs to supply a single equation which can enter the system only as an additional jump condition. This jump condition, however, cannot be universally applied at both discontinuities moving with the 
speeds $D_{s}$ and $D_{p}$, because it would lead to an over-determined system. We must therefore distinguish between the two types of transitions: $w_{0} \rightarrow w_{+}$and $w_{+} \rightarrow w_{-}$.

Consider the simplest isothermal case first. As we have already seen, neither of the discontinuities can be constrained through the entropy criterion, so one needs to search for more subtle restrictions.

Notice that the first discontinuity $w_{0} \rightarrow w_{+}$, which we call a shock, satisfies the Lax criterion (Lax , 1971)

$$
c_{t_{0}} \geq D \geq c_{t_{+}}
$$

where $c_{t}$ is given by (2.2.2), and $D=\sqrt{\left(\sigma_{0}-\sigma_{+}\right) /\left(w_{0}-w_{+}\right)}$is the velocity of the shock. As a result of (2.4.1) the local configuration of characteristics around the jump discontinuity consists of three characteristics coming (from the "past") and one characteristic leaving (to the "future"). This implies stable interaction of the discontinuity with acoustic waves. On the contrary the second transition $w_{+} \rightarrow w_{-}$, which we call a kink (known also as non-evolutionary or under-compressive shock), violates the Lax criterion since $c_{t_{-}} \geq D$ and $c_{t_{+}} \geq D$. In this case two characteristics are coming to the discontinuity and two are leaving. This results in an instability unless an additional jump condition is prescribed. The former arguments can be easily extended to the adiabatic case with the substitution of $c_{s}$ for $c_{t}$ and appropriate adjustments for the number of characteristics.

Our analysis so far has been based exclusively on the mathematical structure of the equations. The principal physical difference between shocks and kinks can be illustrated by the following argument. Consider for simplicity an isothermal model with a generic discontinuity propagating with a constant velocity $D>0$ and transforming a configuration with strain $w_{+}$into a configuration with strain $w_{-}$. Assume for simplicity of argument that the Rankine-Hugoniot conditions (2.1.8) are satisfied not only for the final configuration $w_{-}$but also for every intermediate state $w$ inside the transition region. Under this assumption, we certainly neglect important physical mechanisms of dissipative and dispersive nature inside the transition zone; a more careful analysis, however, does not affect the main conclusion. Along the chosen trajectory inside the transition front one can calculate the "microscopic" rate of the total energy loss (gain). In particular, for the process which begins at $w_{+}$and ends at the current $w$, the rate of dissipation equals

$$
\mathfrak{R}\left(w, w_{+}\right)=D\left(f\left(w_{+}\right)-f(w)+1 / 2\left(v_{+}^{2}-v^{2}\right)\right)+\left(\sigma_{+} v_{+}-\sigma v\right)
$$

The corresponding release of the total energy $\psi\left(w, w_{+}\right)=-\mathfrak{R} / D$ can now be computed explicitly. We obtain

$$
\psi\left(w, w_{+}\right)=f(w)-f\left(w_{+}\right)-\left(\sigma_{+}+1 / 2 \frac{\sigma_{+}-\sigma_{-}}{w_{+}-w_{-}}\left(w-w_{+}\right)\right)\left(w-w_{+}\right) .
$$


As one can expect from (2.1.10), at $w=w_{-}$, we obtain

$$
\psi\left(w_{-}, w_{+}\right)=-\left(f\left(w_{+}\right)-f\left(w_{-}\right)-1 / 2\left(\sigma_{+}+\sigma_{-}\right)\left(w_{+}-w_{-}\right)\right) .
$$

The schematic graphs of $\psi\left(w, w_{+}\right)$, based on (2.4.3), are shown in Fig. 5 separately for the shocks and for the kinks involved in the solution (2.3.6).

Notice that in the case of shocks, the energy decreases monotonically, while in the case of kinks, there exists a finite energy barrier. The propagation of the kink is therefore associated with the "barrier crossing" (see the discussion of the corresponding autocatalytic process in Slepyan (2000, 2001) and Puglisi and Truskinovsky (2001)). In spite of the nonzero dissipation, this process does not require extra energy from outside, although it must be sustained by the forward energy transfer from behind the kink to its front. The availability of such a mechanism depends on the presence in the dispersive spectrum of the associated micromodel of the waves whose group velocity is larger than the phase velocity. Obviously, the velocity of the kink must be appropriate to make this (dispersional) "tunneling" possible, which explains the microscopic origin for the macroscopic restriction on the kink's velocity (kinetic relation).

For the adiabatic kinks the most general kinetic relation can be written in the form

$$
\psi\left(w_{+}, w_{-}, v_{+}, v_{-}, T_{+}, T_{-}, D\right)=0
$$

The Rankine-Hugoniot conditions and the Galilean invariance allow one to reduce this formula, at least locally, to a relation among three variables only, e.g.

$$
\bar{\psi}\left(w_{+}, T_{+}, D\right)=0
$$

We remark that in the phenomenological modeling, conditions analogous to (2.4.6) are often formulated in terms of a relation between two variables: the configurational force $G \equiv-[S]$ (or $-[S]\{T\}$ ) and the conjugate "flux" $D$.

\subsection{Nucleation}

In order to understand better what happens when we select a nucleation point $x=x_{0}$, consider the behavior of the self-similar solutions (2.3.6) and (2.3.7) after sufficiently small time $t=\Delta t$. For simplicity, we shall limit our discussion to the isothermal case.

It will be convenient to parameterize functions $w(x, \Delta t)$ and $v(x, \Delta t)$ representing solution (2.3.6) at $t=\Delta t$ by $x$ and present them in the $(w, v)$ plane. One can see that through this construction, we obtain a set of points describing the piece-wise constant solution of the self-similar problem which can be connected to form a loop, beginning and ending at the point $\left(w_{0}, v_{0}=0\right)$ (see Fig. 6). The detailed configuration of the connecting segments depends on the fine internal structure of both shocks and kinks: this information obviously lies outside the scope of the non-regularized macro-model. We 
also notice that due to the self-similarity of the solution, the resulting loop does not alter as $\Delta t \rightarrow 0$, even though both strain and velocity fields converge to the constant values everywhere outside the point $x=x_{0}$.

From the above observations one can conclude that by selecting a nucleation point $x_{0}$, we have actually supplemented our constant initial data with a singular part represented by a measure in the configurational space $(w, v)$ localized at $x=x_{0}$. Since the macroscopic energy of this measure-valued nucleus is identically zero, the integral contribution to the initial data should be measured by the corresponding energy density, which is finite and which can be used as a measure of stability of the metastable state. The presence of the actual barrier separating the uniform initial state and the state with the superimposed loop can only be made explicit in the framework of a regularized model, which contains some finite internal length scale.

Finally we remark that based on the singular initial data presented above one can compute the instantaneous rate of dissipation $\mathfrak{R}$

$$
\Re=D_{1}([f]-\{\sigma\}[w])_{\text {shock }}+D_{2}([f]-\{\sigma\}[w])_{\text {kink }} .
$$

If the kinetic relation is known, the energy release rate (2.5.1), which, due to the selfsimilarity of the solution, does not depend on $t$, can be calculated as a function of $w_{0}$; the fact that $\mathfrak{R} \neq 0$ at $t=0$ means that the initial data (2.3.5) are instantly "dissipative."

\section{The regularized model}

In this section, we introduce a regularization of our model, which combines the simplest gradient correction to the elastic energy with the Fourier heat conductivity and Kelvin viscoelasticity (Slemrod (1984a), Truskinovsky (1985, 1993b), Turteltaub (1997a, b), Ngan and Truskinovsky (1999)). For brevity we shall call this model thermo-viscocapillary (TVC) model. Since after this regularization the group velocity of plane waves can be larger than the phase velocity one can conclude that the TVC model may in principle provide a mechanism for the barrier crossing inside the subsonic kinks.

\subsection{Governing equations}

Consider a a thermo-elastic material whose energy depends on both strain and strain gradient $e=e(w, \partial w / \partial x, s)$. Then, instead of (2.1.1), we obtain

$$
\frac{\partial w}{\partial t}=\frac{\partial v}{\partial x}, \quad \frac{\partial v}{\partial t}=\frac{\partial}{\partial x}\left(\sigma-\frac{\partial m}{\partial x}\right), \quad \frac{\partial e}{\partial t}=\sigma \frac{\partial v}{\partial x}+m \frac{\partial^{2} v}{\partial x^{2}}
$$

Here $\sigma=\partial e / \partial w$ is the stress, and

$$
m=\frac{\partial e}{\partial(\partial w / \partial x)}
$$


is the hyper-stress (moment). For determinacy we assume that the strain gradient contribution to the energy is of the form

$$
e\left(w, \frac{\partial w}{\partial x}, s\right)=e(w, s)+\varepsilon\left(\frac{\partial w}{\partial x}\right)^{2}
$$

where $\varepsilon$ is a positive constant which characterizes the degree of non-locality. Then for the hyper-stress, we obtain $m=2 \varepsilon \partial w / \partial x$.

The dissipative part of our regularized model includes Fourier heat conductivity and Kelvin viscosity. Specifically, introduce a heat flux

$$
q=-\kappa \frac{\partial T}{\partial x}
$$

with $\kappa$ being the coefficient of heat conductivity, and rewrite the energy equation as

$$
\frac{\partial e}{\partial t}=\sigma \frac{\partial v}{\partial x}+m \frac{\partial^{2} v}{\partial x^{2}}-\frac{\partial q}{\partial x} .
$$

The viscous contribution to the stress takes a conventional form

$$
\sigma \rightarrow \sigma+\eta \frac{\partial v}{\partial x}
$$

where $\eta$ is the viscosity coefficient. With these additional assumptions, the system (3.1.1) takes the form

$$
\begin{gathered}
\frac{\partial w}{\partial t}=\frac{\partial v}{\partial x}, \quad \frac{\partial v}{\partial t}=\frac{\partial}{\partial x}\left(\sigma(w, s)-2 \varepsilon \frac{\partial^{2} w}{\partial x^{2}}+\eta \frac{\partial v}{\partial x}\right) \\
\frac{\partial}{\partial t}\left(e(w, s)+\varepsilon\left(\frac{\partial w}{\partial x}\right)^{2}+\frac{1}{2} v^{2}\right)=\frac{\partial}{\partial x}\left(\left(\sigma(w, s)-2 \varepsilon \frac{\partial^{2} w}{\partial x^{2}}+\eta \frac{\partial v}{\partial x}\right) v\right) \\
+2 \varepsilon \frac{\partial}{\partial x}\left(\frac{\partial v}{\partial x} \frac{\partial w}{\partial x}\right)+\kappa \frac{\partial^{2} T}{\partial x^{2}} .
\end{gathered}
$$

The equations (3.1.6) constitute the basis of the TVC model. We remark that the energy equation (3.1.6. ) can be substituted by an equivalent equation governing the balance of entropy

$$
T \frac{\partial s}{\partial t}=\kappa \frac{\partial^{2} T}{\partial x^{2}}+\eta\left(\frac{\partial v}{\partial x}\right)^{2} .
$$




\subsection{Material model}

In order to be able to perform numerical simulations, we need to specify at least one of the thermodynamic potentials, for instance, $f(w, T)$. We make the simplest assumptions:

(i) the isothermal stress-strain curve is cubic;

(ii) the stress at constant strain is a linear function of temperature;

(iii) the equilibrium (Maxwell) strains $w_{1}$ and $w_{2}$ are independent of temperature.

These assumptions lead to the following stress-strain relation

$$
\sigma(w, T)=A+B T+K\left(w-w_{1}\right)\left(w-w_{2}\right)\left(w-1 / 2\left(w_{1}+w_{2}\right)\right)
$$

where $w_{1}, w_{2}>w_{1}, A, B$ and $K$ are all positive constants. One can show that in this model the transformation strain $w_{2}-w_{1}$ is indeed independent of temperature, that the Maxwell stress increases linearly with temperature

$$
\sigma_{M}=A+B T
$$

and that the latent heat of transformation from the low-strain phase to the high strain phase is negative (see below). We remark that these three properties are in qualitative agreement with the experimental data on various shape memory alloys (see, for instance, Leo et al. (1993), and Shield et al. (1997)).

By integrating the stress-strain relation, we obtain the expression for the internal energy

$$
\begin{gathered}
e(w, T)=A w+K\left\{\frac{1}{4} w^{4}-\frac{1}{2}\left(w_{1}+w_{2}\right) w^{3}+\frac{1}{2}\left(\frac{1}{2} w_{1}^{2}+2 w_{1} w_{2}+\frac{1}{2} w_{2}^{2}\right) w^{2}-\frac{1}{2} w_{1} w_{2}\left(w_{1}+w_{2}\right) w\right\} \\
+\varphi(T) .
\end{gathered}
$$

Following common practice we shall assume that the specific heat $C_{V}$ is constant. Then

$$
\varphi(T)=C_{V}\left(T-T_{r}\right)
$$

where $T_{r}$ is some reference temperature. The free energy can now be written as

$$
\begin{gathered}
f(w, T)=A w+B T w+ \\
K\left\{\frac{1}{4} w^{4}-\frac{1}{2}\left(w_{1}+w_{2}\right) w^{3}+\frac{1}{2}\left(\frac{1}{2} w_{1}^{2}+2 w_{1} w_{2}+\frac{1}{2} w_{2}^{2}\right) w^{2}-\frac{1}{2} w_{1} w_{2}\left(w_{1}+w_{2}\right) w\right\} \\
-C_{V} T \ln \left(T / T_{r}\right) .
\end{gathered}
$$

To demonstrate the physical meaning of the parameter $B$, one can compute the specific entropy, 


$$
s=-B w+C_{V} \ln \left(T / T_{r}\right)+C_{V}
$$

and the latent heat

$$
Q=T\left(s_{2}-s_{1}\right)=-B\left(w_{2}-w_{1}\right) T
$$

One can see that for $B>0$ the heat is released when the material transforms from the low strain phase to the high strain phase. This in turn implies that the equilibrium phase boundary in the stress-temperature phase diagram has a positive slope: $d \sigma_{M} / d T=B \geq 0$.

\subsection{Non-dimensionalization}

In this sub-section, we normalize the variables and introduce the main dimensionless parameters of the problem. Define

$$
x=l_{r} \hat{x}, t=t_{r} \hat{t}, T=T_{r} \hat{T}, \sigma=\sigma_{r} \hat{\sigma}, e=e_{r} \hat{e}, f=f_{r} \hat{f}, s=s_{r} \hat{s}, v=\left(l_{r} / t_{r}\right) \hat{v}
$$

where the reference scales $l_{r}, t_{r}, T_{r}, \sigma_{r}, e_{r}, f_{r}$ and $s_{r}$ are chosen in such a way that

$$
\sigma_{r}=e_{r}=f_{r}=\left(l_{r} / t_{r}\right)^{2}=s_{r} T_{r}=C_{V} T_{r}=K=A
$$

This specific choice implies that all energy scales are of the same order and that the maximum size of the hysteresis is of the order of the Maxwell stress. We choose the length scale to be of the order of the capillary length

$$
l_{r}^{2}=\varepsilon / A
$$

Finally, we assume that the transformation strain is of order one, and, for determinacy, we choose equilibrium strains to be

$$
w_{1}=0, w_{2}=1
$$

With these assumptions, we can now non-dimensionalize the main system of equations. In dimensionless variables, the system (3.1.6) can be rewritten as

$$
\begin{aligned}
& \frac{\partial^{2} u}{\partial t^{2}}=\frac{\partial \sigma(w, t)}{\partial x}+\mathbf{W}_{1} \frac{\partial^{3} u}{\partial x^{2} \partial t}-\frac{\partial^{4} u}{\partial x^{4}} \\
& T \frac{\partial s(w, T)}{\partial t}=\mathbf{W}_{2}^{-1} \frac{\partial^{2} T}{\partial x^{2}}+\mathbf{W}_{1} \frac{\partial^{2} u}{\partial x \partial t}
\end{aligned}
$$

where $w=\partial u / \partial x$ and the two constitutive functions $\sigma(w, T)$ and $s(w, t)$ are specified by the formulas 


$$
\begin{gathered}
\sigma(w, T)=1+\mathbf{W}_{3} T+w(w-1)(w-1 / 2), \\
s(w, T)=-\mathbf{W}_{3} w+\ln T+1
\end{gathered}
$$

The non-dimensional forms of the other important thermodynamic functions are presented below

$$
\begin{aligned}
& e(w, T)=w+1 / 4 w^{4}-1 / 2 w^{3}+1 / 4 w^{2}+T-1, \\
& f(w, T)=w+\mathbf{W}_{3} w T+\frac{1}{4} w^{4}-\frac{1}{2} w^{3}+\frac{1}{4} w^{2}-T \ln T
\end{aligned}
$$

To summarize, the system of equations $(3.3 .5,3.3 .6)$ contains three essential nondimensional parameters $\mathbf{W}_{1}, \mathbf{W}_{2}$, and $\mathbf{W}_{3}$ :

$$
\mathbf{W}_{1}=\frac{\eta}{\sqrt{\varepsilon}}, \quad \mathbf{W}_{2}=\frac{C_{V} \sqrt{\varepsilon}}{\kappa}, \quad \mathbf{W}_{3}=\frac{B}{C_{V}}
$$

The first parameter, $\mathbf{W}_{1}$, can be viewed as a dimensionless ratio of viscous dissipation and interfacial energy. The second parameter, $\mathbf{W}_{2}$, represents a dimensionless measure of the interfacial energy over the heat conductive dissipation. Finally, parameter $\mathbf{W}_{3}$ has a purely thermodynamical nature, and can be considered as a dimensionless expression of the heat of transformation in the units of specific heat.

\section{Traveling waves}

In this section, we briefly review the travelling wave solutions to the system (3.3.5, 3.3.6) describing the internal structure of a moving kink and explicitly compute the kinetic relation in a range of parameters $\mathbf{W}_{1}, \mathbf{W}_{2}$ and $\mathbf{W}_{3}$. We refer the reader to Ngan and Truskinovsky (1999) for additional details.

\subsection{Boundary value problem}

Consider a special class of solutions to the system $(3.3 .5,3.3 .6)$ in the form of the travelling waves $w=w(z), v=v(z), T=T(z)$, where $z=x-D t$, and $D$ is the wave velocity. After the variable $v$ is eliminated, the main system of non-dimensional equations can be written in the form

$$
\begin{gathered}
w^{\prime \prime}=1 / 2\left\{\sigma(w, T)-\sigma\left(w_{+}, T_{+}\right)-\mathbf{W}_{1} D w^{\prime}-D^{2}\left(w-w_{+}\right)\right\} \\
T^{\prime}=-D \mathbf{W}_{2}\left\{e(w, T)-e\left(w_{+}, T_{+}\right)-1 / 2 D^{2}\left(w-w_{+}\right)^{2}-w^{\prime 2}-\sigma\left(w_{+}, T_{+}\right)\left(w-w_{+}\right)\right\}
\end{gathered}
$$


where $e(w, T)$ and $\sigma(w, T)$ are given by (3.3.7, 3.3.9). Equations (4.1.1, 4.1.2) together with the constitutive relations $(3.3 .7,3.3 .9)$ and the boundary conditions

$$
w( \pm \infty)=w_{ \pm} \text {and } T( \pm \infty)=T_{ \pm}
$$

constitute a boundary value problem on the real axis. The desired solution corresponds to the heteroclinic trajectory of the dynamical system $(4.1 .1,4.1 .2)$, and the main problem is to find restrictions on the set of boundary values $w_{ \pm}, v_{ \pm}, T_{ \pm}$and $D$ which guarantee the existence of such a trajectory. After the solution is known, the rate of entropy production (and the kinetic relation) can be calculated explicitly from

$$
-D[s]=\int_{-\infty}^{\infty} \frac{T^{\prime 2}}{\mathbf{W}_{2} T^{2}}+\frac{\mathbf{W}_{1} v^{\prime 2}}{T} .
$$

Suppose that the state in front of the discontinuity is given. This fixes one of the critical points of the system $(4.1 .1,4.1 .2)$, and leaves the other critical point unspecified until the speed of the jump discontinuity $D$ is prescribed. Now, the problem of admissibility can be viewed as a nonlinear eigenvalue problem with respect to $D$. The fact that kinks correspond to saddle-to-saddle trajectories, while shocks correspond to saddle-to-node (focus) trajectories, is responsible for the difference in the number of admissibility conditions. Since the saddle-to-node transition is structurally stable, the spectrum of the admissible speeds for the shocks will be continuous. On the other hand, since the saddle-to-saddle transition is not structurally stable, one obtains in the case of kinks a discrete set of admissible velocities $D$ selected by what became known as kinetic relation.

\subsection{Kinetic relation}

In the simplest special case $\mathbf{W}_{3}=0, C_{V}=\infty$, the temperature can be completely eliminated from (4.1.1) and the problem reduces to a purely mechanical one. The advantage of this simplified setting (isothermal regime) is that one can construct a closed form solution describing kinks

$$
w(z)=\frac{w_{+}+w_{-}}{2}+\frac{w_{+}-w_{-}}{2} \tanh \left\{\frac{w_{+}-w_{-}}{4}\left(z-z_{0}\right)\right\} .
$$

It is straightforward to check that the ansatz (4.2.1) is compatible with the equation (4.1.1) if parameters $w_{-}, w_{+}$and $D$ satisfy the following relations

$$
\begin{gathered}
\left(w_{-}-w_{+}\right)^{2}+3\left(1-12 / \mathbf{W}_{1}^{2}\right)\left(w_{+}+w_{-}-1\right)^{2}=1, \\
D=3\left(w_{+}+w_{-}-1\right) / \mathbf{W}_{1},
\end{gathered}
$$

which describe points of the discrete spectrum of the eigenvalue problem (4.1.1-4.1.3). With parameter $w_{-}$eliminated from (4.2.2) the corresponding pairs $\left(w_{+}, D\right)$ describe a 
one-dimensional subset of kinks inside the full admissibility set describing all possible traveling waves (shocks and kinks) for the given value of $\mathbf{W}_{1}$.

The generic picture is shown in Fig. 7. Notice that all kinetic curves originate from the point $A$, which marks the onset of metastability in the isothermal problem (see (2.2.1)), and end on the sonic line (e.g. point $O$ for $\mathbf{W}_{1}=2.5$ ). Shocks occupy a $2 \mathrm{D}$ subset of the supersonic domain. For instance, at $\mathbf{W}_{1}=2.5$, the admissible region consists of the curve $A O$ (kinks) and the shaded area $D O A^{\prime}$ (shocks) ${ }^{2}$. The kinetic curves for the adiabatic case $\left(\mathbf{W}_{2}=\infty\right)$ possess the same basic features, with the only difference that the isothermal sonic line $D=c_{t}$ has to be substituted by the adiabatic sonic line $D=c_{s}$, and that the point $A$ has to be replaced by the corresponding adiabatic limit of metastability (2.2.3).

In the general case which is neither isothermal, nor adiabatic (see Fig. 8), all kinetic curves originate from a particular point $M$ whose location may be found explicitly (see Ngan and Truskinovsky (1999)). A close look at the structure of the kinetic curves around point $M$ reveals rather complicated behavior. For example, at $\mathbf{W}_{2}=40$ one can see that there exist multiple solutions. These solutions are characterized by finite oscillations of strain in the transitional region which hints towards mixing of the two phases within the phase boundary structure; the number of the phase switchings increases as we go from point $P$, to $Q$, and then to $R$. Stability of these solutions with multiple oscillations is highly questionable and in the rest of the paper we shall only consider traveling waves with the monotone structure.

\subsection{Mobility curves}

As mentioned in Section 2, the kinetic relation is often formulated in terms of a relation between the configurational force $G$ and the conjugate "flux" $D$. The kinetic curves represented in these coordinates are often called mobility curves. To obtain the mobility curves in our case we must fix one of the parameters, which we choose to be the temperature ahead of the discontinuity. The computed $\mathbf{W}_{2}$ dependence of the mobility curves is shown in Fig. 9. One can see that mobility curves, corresponding to different values of $\mathbf{W}_{2}$, converge to point $M$ as $D \rightarrow 0$ (same as point $M$ in Fig. 8). The configurational force $G$ at this point is different from zero, which means that the dissipative potential at zero velocities is not smooth (trapping). At large $\mathbf{W}_{2}$ (small heat conductivity), the mobility curves become non-monotone; a closer look around point $M$ again reveals complex loop structures which we shall neglect in the rest of the paper.

Overall our analysis reveals two important effects distinguishing adiabatic kinks from their isothermal counterparts. First, in the adiabatic case, the mobility curves do not originate from the point where the driving force is zero due to the negative feedback provided by the latent heat. The second effect is the multi-valuedness and nonmonotonicity of the mobility curves at low velocities, which in principle can give rise to a stick-slip behavior (see, for instance, Rosakis and Knowles (1997)).

\footnotetext{
${ }^{2}$ The reader is referred to Truskinovsky (1994) and Ngan and Truskinovsky (1999) for the reasoning why not all supersonic shocks are admissible and for the explicit construction of the curve $\mathrm{OA}^{\prime}$.
} 


\section{Solution of the Riemann problem}

With the location of the nucleation point fixed and kinetic relation specified, one can close the system of equations describing self-similar decomposition of a metastable state. In this Section we explicitly determine parameters of the flow including velocities of the precursor shocks and of the trailing subsonic kinks. As before, we consider isothermal and adiabatic cases separately.

\subsection{Isothermal case}

Consider a metastable state characterized by the constant temperature $T=T_{0}$, constant strain $w=w_{0}$, and zero velocity $v=v_{0}=0$. To determine numerical values of parameters of the nontrivial self-similar solution, one must simultaneously solve the following equations:

$$
\begin{aligned}
& -D_{s}\left(w_{0}-w_{+}\right)=\left(v_{0}-v_{+}\right), \\
& -D_{s}\left(v_{0}-v_{+}\right)=\sigma\left(w_{0}, T_{0}\right)-\sigma\left(w_{+}, T_{0}\right),
\end{aligned}
$$

for the shock, and

$$
\begin{aligned}
& -D_{p}\left(w_{+}-w_{-}\right)=\left(v_{+}-v_{-}\right) \\
& -D_{p}\left(v_{+}-v_{-}\right)=\sigma\left(w_{+}, T_{0}\right)-\sigma\left(w_{-}, T_{0}\right)
\end{aligned}
$$

for the kink. In addition, we require that $v_{0}=0$ and $v_{2}=0$. By imposing the constitutive model from section 4.2, we obtain the following additional jump condition for the kink (see (4.2.2))

$$
\left(w_{+}-w_{-}\right)^{2}+3\left(1-12 / \mathbf{W}_{1}^{2}\right)\left(w_{-}+w_{+}-1\right)^{2}=1 .
$$

With five equations (5.1.1-5.1.5) for the five unknowns $D_{s}, D_{p}, w_{1}, w_{2}, v_{1}$ the algebraic problem is well defined. Notice that our solution depends on the parameters of the regularization only through the non-dimensional ratio $\mathbf{W}_{1}$. The numerical solutions at different values of $\mathbf{W}_{1}$ are illustrated in Fig. 10. One can see that in the isothermal problem the self-similar solution describing nucleation is unique.

We remark that the computed solution describes an "explosion" - the energy release due to the decomposition of a metastable state. It is then of interest to determine how this energy is distributed. A fraction of the released energy will be dissipated inside shocks and kinks. The rest will be transformed into kinetic energy of the moving material. The overall rate of dissipation is equal to 


$$
E^{\prime}=2\left\{D_{s} f\left(w_{0}\right)-D_{p} f\left(w_{-}\right)-\left(D_{s}-D_{p}\right)\left(f\left(w_{+}\right)+\frac{v_{+}^{2}}{2}\right)\right\} .
$$

Inside the shocks the energy is dissipated with the rate

$$
A_{s}=2 D_{s}\left\{\int_{w_{+}}^{v_{0}} \sigma d w-\frac{\sigma_{0}+\sigma_{+}}{2}\left(w_{0}-w_{+}\right)\right\} .
$$

Similarly, inside the kinks the rate of dissipation is

$$
A_{p}=2 D_{p}\left\{\int_{w_{-}}^{w_{+}} \sigma d w-\frac{\sigma_{+}+\sigma_{-}}{2}\left(w_{+}-w_{-}\right)\right\} .
$$

Obviously $E^{\prime}=A_{s}+A_{p}$. These expressions can be compared with the overall rate of the elastic energy release

$$
E_{e l}^{\prime}=2 D_{s}\left[f\left(w_{0}\right)-f\left(w_{+}\right)\right]+2 D_{p}\left[f\left(w_{+}\right)-f\left(w_{-}\right)\right] .
$$

In particular one can compute the fractions of the released energy dissipated in the unit of time by shocks $\left(x_{1}\right)$ and kinks $\left(x_{3}\right)$ and the fraction transformed into the kinetic energy $\left(x_{2}\right)$. From the expressions $(5.1 .7,5.1 .8,5.1 .9)$ we obtain

$$
\begin{gathered}
x_{1}=\frac{D_{s}\left[f\left(w_{0}\right)-f\left(w_{+}\right)-\frac{\sigma\left(w_{0}\right)+\sigma\left(w_{+}\right)}{2}\left(w_{0}-w_{+}\right)\right]}{D_{s}\left[f\left(w_{0}\right)-f\left(w_{+}\right)\right]+D_{p}\left[f\left(w_{+}\right)-f\left(w_{-}\right)\right]}, \\
x_{2}=\frac{\left(D_{s}-D_{p}\right) \frac{v_{+}^{2}}{2}}{D_{s}\left[f\left(w_{0}\right)-f\left(w_{+}\right)\right]+D_{p}\left[f\left(w_{+}\right)-f\left(w_{-}\right)\right]}, \\
x_{3}=\frac{D_{p}\left[f\left(w_{+}\right)-f\left(w_{-}\right)-\frac{\sigma\left(w_{+}\right)+\sigma\left(w_{-}\right)}{2}\left(w_{+}-w_{-}\right)\right]}{D_{s}\left[f\left(w_{0}\right)-f\left(w_{+}\right)\right]+D_{p}\left[f\left(w_{+}\right)-f\left(w_{-}\right)\right]} .
\end{gathered}
$$

The computational results are presented in Fig 11. Notice that when $\mathbf{W}_{1}$ tends to zero, the percentage of the energy dissipated inside the kinks approaches zero while that in the shock waves remains finite. 


\subsection{Adiabatic case}

Now consider the adiabatic problem. As we have seen in Section 2 the dynamic solution in this case consists again of two symmetric shocks emerging from the nucleation point and propagating into opposite directions. These shocks are followed by two kinks (see Fig. 4). The initial configuration is of the form $w=w_{0}, v=v_{0}=0$ with $T=T_{0}$, for some fixed dimensionless temperature $T_{0}$. The Rankine-Hugoniot jump conditions across the shock take the form

$$
\begin{gathered}
-D_{s}\left(w_{0}-w_{+}\right)=\left(v_{0}-v_{+}\right), \\
-D_{s}\left(v_{0}-v_{+}\right)=\sigma\left(w_{0}, T_{0}\right)-\sigma\left(w_{+}, T_{+}\right), \\
-D_{s}\left\{e\left(w_{0}, T_{0}\right)+\frac{v_{0}^{2}}{2}-e\left(w_{+}, T_{+}\right)-\frac{v_{+}^{2}}{2}\right\}=\sigma\left(w_{0}, T_{0}\right) v_{0}-\sigma\left(w_{+}, T_{+}\right) v_{+} .
\end{gathered}
$$

Across the kink we obtain

$$
\begin{gathered}
-D_{p}\left(w_{+}-w_{-}\right)=\left(v_{+}-v_{-}\right) \\
-D_{p}\left(v_{+}-v_{-}\right)=\sigma\left(w_{+}, T_{+}\right)-\sigma\left(w_{-}, T_{-}\right), \\
-D_{p}\left\{e\left(w_{+}, T_{+}\right)+\frac{v_{+}^{2}}{2}-e\left(w_{-}, T_{-}\right)-\frac{v_{-}^{2}}{2}\right\}=\sigma\left(w_{+}, T_{+}\right) v_{+}-\sigma\left(w_{-}, T_{-}\right) v_{-} .
\end{gathered}
$$

As in the isothermal case, here we also assume that $v_{0}=0$ and $v_{-}=0$. Given the initial state $\left(w_{0}, T_{0}\right)$, we have six relations (5.2.1-5.2.6) for the seven unknowns $D_{s}, D_{p}, w_{1}, w_{2}, T_{1}, T_{2}, v_{1}$. The admissibility criterion implicitly formulated in Section 4 gives us an extra condition to close the system; formally, the kinetic relation can be expressed in the form $\widetilde{\psi}\left(w_{1}, T_{1}, w_{2}\right)=0$ where the specific form of the function $\widetilde{\psi}$ depends on the nondimensional parameters $\mathbf{W}_{1}, \mathbf{W}_{2}$ and $\mathbf{W}_{3}$. By solving the above equations one can completely specify the self-similar dynamical process which follows the nucleation event.

Our numerical results are presented in Fig. 12, where we fixed parameters $\mathbf{W}_{1}, \mathbf{W}_{3}$ and $T_{0}$ and varied parameter $\mathbf{W}_{2}$. By comparing the adiabatic solutions with the similar solutions in the isothermal case, we observe a new feature: for sufficiently large $\mathbf{W}_{2}$, the initial problem can have two nontrivial solutions. Thus, for instance, at $\mathbf{W}_{2}=40$, and $w_{0}$ between 0.083 and 0.123 , our system of algebraic equations generates two values of $w_{+}$. The nonuniqueness here results from the nonmonotonicity of the mobility curves (see Fig. 9); it should be noted that these two solutions arise from the "main branch" of the kinetic relation, not the "loop structure", which have been excluded from the analysis. In order to decide which of the two self-similar solutions is an attractor in the non-selfsimilar setting, one needs to solve the regularized initial value problem with a finite localized perturbation added to the original Riemann data. 


\section{Numerical solutions}

In this section we look closely at the initial stage of the nucleation event and employ the TVC model from Section 4 to study the non-self-similar stage of the nucleation process. Technically, we need to solve numerically equations $(3.3 .5,3.3 .6)$ in the infinite domain for initial data with a small but finite support.

\subsection{Isothermal case}

Our method of spatial discretization was motivated by the idea of an infinite onedimensional discrete chain with masses connected by nonlinear springs. In the long wave limit such a chain can be described by continuum with energy density depending on both strains and strain gradients. One can show that the corresponding gradient model will be characterized by a non-negative capillarity coefficient $(\varepsilon>0)$ only if the interaction of both first and second nearest neighbors is taken into consideration and only if the interaction of the second nearest neighbor is unstable (e.g. [Mindlin (1965), Kunin (1982), Triantafyllidis and Bardenhagen (1993)).

Define $u_{n}$ as the displacement of the $n$th particle from its reference position, and $a$ as the reference length. Then the elastic energy of the chain with nearest and next to nearest neighbor interactions can be written in the form

$$
W=\sum_{n} a f_{1}\left(\frac{u_{n+1}-u_{n}}{a}\right)+2 a f_{2}\left(\frac{u_{n+2}-u_{n}}{2 a}\right) .
$$

The springs connecting nearest and next to nearest neighbors are characterized by elastic potentials $f_{1}(w)$ and $f_{2}(w)$, accordingly. We remark that the reference states for the two springs may be different, and that the corresponding spring stiffnesses at zero force may have different signs; a finite chain of this type with quadratic potentials $f_{1}$ and $f_{2}$ has been recently studied in Charlotte and Truskinovsky (2001).

The kinetic energy of the chain is given by the formula

$$
K=\frac{1}{2} \sum_{n} a\left(\frac{\partial u_{n}}{\partial t}\right)^{2}
$$

where the scaling is chosen to be compatible with the assumption that the reference density is equal to unity. The equation of motion generated by (6.1.1-2) takes the form

$$
\frac{\partial^{2} u_{n}}{\partial t^{2}}=f_{1}^{\prime}\left(\frac{u_{n+1}-u_{n}}{a}\right)+f_{2}^{\prime}\left(\frac{u_{n+2}-u_{n}}{2 a}\right)-f_{1}^{\prime}\left(\frac{u_{n}-u_{n-1}}{a}\right)-f_{2}^{\prime}\left(\frac{u_{n}-u_{n-2}}{2 a}\right) .
$$

To obtain a long wave continuum limit of (6.1.1-3), one can replace the finite differences by the first few terms of the Taylor expansion, and substitute the finite sums by integrals. Then for the elastic and kinetic energies we obtain 


$$
W=\int \hat{f}\left(u_{x}\right)+\varepsilon u_{x x}^{2}, K=\frac{1}{2} \int u_{t}^{2},
$$

where the sub-index denotes partial derivative in $x$ while the superimposed dot denotes partial derivative in time. In (6.1.4) the following new functions were introduced

$$
\begin{aligned}
& \hat{f}\left(u_{x}\right)=f_{1}\left(u_{x}\right)+2 f_{2}\left(u_{x}\right), \\
& \varepsilon\left(u_{x}\right)=-a^{2}\left\{f_{1}^{\prime}\left(u_{x}\right) / 24+f_{2}^{\prime}\left(u_{x}\right) / 3\right\} .
\end{aligned}
$$

The continuum equation of motion can now be written in the form

$$
u_{t t}=\left\{\hat{f}^{\prime}\left(u_{x}\right)-2 \varepsilon\left(u_{x}\right) u_{x x x}-\varepsilon^{\prime}\left(u_{x}\right) u_{x x}^{2}\right\}_{x}
$$

It is not hard to check that this regularized wave equation is compatible with the gradient part of our TVC model if

$$
\begin{aligned}
& \hat{f}^{\prime}\left(u_{x}\right)=\sigma\left(u_{x}\right), \\
& \varepsilon\left(u_{x}\right) \equiv \varepsilon=\text { const }
\end{aligned}
$$

For the consistency of this discretization procedure we need to choose functions $f_{1}$ and $f_{2}$ in such a way that (6.1.8-9) are satisfied. Since in our continuous model, $\sigma\left(u_{x}\right)$ was taken to be cubic, it is natural to assume that the functions $f_{1}^{\prime}$ and $f_{2}^{\prime}$ are also cubic polynomials

$$
f_{1}^{\prime}=d_{1}+2 a_{1} u+3 b_{1} u^{2}+4 c_{1} u^{3}, \quad f_{2}^{\prime}=d_{2}+2 a_{2} u+3 b_{2} u^{2}+4 c_{2} u^{3} .
$$

Now, if we choose

$$
\begin{gathered}
a_{1}=\frac{K}{3}+2 c, a_{2}=-\frac{K}{24}-c, b_{1}=-\frac{2 K}{3}, b_{2}=\frac{K}{12}, \\
c_{1}=\frac{K}{3}, c_{2}=-\frac{K}{24}, d_{1}=\frac{4 A}{3}, d_{2}=-\frac{A}{6},
\end{gathered}
$$

where constants $A$ and $K$ are the same as in (3.2.1), and substitute (6.1.10) and (6.1.11) into equations (6.1.5-6), we obtain the correspondence

$$
\sigma\left(u_{x}\right)=A+K\left(u_{x}-1\right)\left(u_{x}-\frac{1}{2}\right) u_{x}, \varepsilon=\frac{1}{2} c a^{2}
$$

Finally, the discrete and continuum models will match if we choose $c=2 \varepsilon / a^{2}$. To simulate viscous damping, one can include into the finite difference equation two additional dissipative terms depending on $\dot{u}_{n}(t)$. We obtain 


$$
\begin{aligned}
\ddot{u}_{n}=f_{1}^{\prime}\left(\frac{u_{n+1}-u_{n}}{a}\right) & +f_{2}^{\prime}\left(\frac{u_{n+2}-u_{n}}{2 a}\right)-f_{1}^{\prime}\left(\frac{u_{n}-u_{n-1}}{a}\right)-f_{2}^{\prime}\left(\frac{u_{n}-u_{n-2}}{2 a}\right) \\
& +\eta a\left(\frac{\dot{u}_{n+1}-\dot{u}_{n}}{a}\right)-\eta a\left(\frac{\dot{u}_{n}-\dot{u}_{n-1}}{a}\right) .
\end{aligned}
$$

Here $\eta$ is the damping coefficient, which we choose to be identical with our viscosity coefficient in (3.1.5). In the long wave approximation (6.1.13) gives

$$
u_{t t}=\left\{\sigma\left(u_{x}\right)-2 \varepsilon u_{x x x}+\eta u_{x t}\right\}_{x}
$$

which is exactly the isothermal adaptation of the TVC equations. In what follows, the system of ODE (6.1.13) will be used as a discretized version of (6.1.15).

\subsection{Isothermal nucleation}

In Section 2, we have found that in the isothermal case there exists a twoparameter family of self-similar solutions describing dynamic decomposition of a metastable state. To single out a unique solution, one had to choose a nucleation point $x=x_{0}$, and specify a non-dimensional parameter $\mathbf{W}_{1}$ (see Section 5). Here instead we study numerically the discrete problem (6.1.13) with the functions $f_{1}$ and $f_{2}$ prescribed in (6.1.10-11) and with parameters $\eta$ and $\varepsilon$ chosen in such a way that $\mathbf{W}_{1}=\eta / \sqrt{\varepsilon}$. We consider a finite chain with a sufficiently large number of points to ignore surface boundary layers (see Charlotte and Truskinovsky (2001)) and simulate the process for sufficiently short time so that the interaction of the radiated waves with the boundary points could be neglected.

In our discrete setting the initial Riemann data considered in Section 2 are approximated by

$$
u_{n}(0)=w_{0} a n, \dot{u}_{n}(0)=0
$$

with strain $w_{0}$ in the metastability interval for the low-strain phase of the material (3.2.1). To initiate the transformation, we need to superimpose to these data a small perturbation centered at $x=0$. In our numerical experiments we considered different types of perturbations, all localized in an interval $-\Delta / 2 \leq x \leq \Delta / 2$, including the sinusoidal type $\delta w=\left(w_{\max }-w_{0}\right) \cos \left(\frac{\pi x}{\Delta}\right)$ and the power law type $\delta w=\left(w_{\max }-w_{0}\right)\left\{1-\left(\frac{x}{\Delta}\right)^{2}\right\}^{\mathrm{n}}$ with $n=2$ and $n=6$. Here, $w_{\max }$ is the maximum strain and $\Delta$ is the spatial extent of the perturbation.

The results of the numerical experiments presented in Fig. 13-15 confirm that large perturbations evolve into a regime approaching the self-similar dynamic solution of Section 5 while small perturbations gradually decay. This is compatible with the existence of two attractors and suggests that the nucleation criterion should be related to 
the size of the domain of attraction of the trivial regime (decay). Specifically, Fig. 13 illustrates a typical solution with nucleation. For the case shown, the perturbation was created at time $t=t_{1}$ and the simulation was terminated at some later time $t=t_{2}$. As expected, two precursor shocks move away from the nucleation site, followed by a pair of slower moving kinks.

To compare the behavior of the discrete and continuum models quantitatively, the experiments were repeated for different values of initial strain $w_{0}$ and different parameters $\mathbf{W}_{1}$. The results for the two models, regularized and non-regularized, are compared in Fig. 14. One can see that semi-analytic and numerical data are practically undistinguishable. Finally, Fig. 15 shows how the magnitude of the critical perturbation $\Delta w=w_{\max }-w_{0}$ varies with the extent and the shape of the perturbation. The dashed region in this figure corresponds to supercritical perturbations, sufficient to initiate a dynamic nucleation.

\subsection{Critical nucleus}

In this subsection, we compute analytically the lower bound for the energy associated with the critical perturbation and compare it with numerical data from Section 6.2. Such a bound can be obtained as an energy of the critical nucleus corresponding to a saddle point (of the static problem) with a one-dimensional unstable manifold. The critical perturbation so defined is then necessarily located on the boundary of the basin of attraction of the initial metastable configuration (e.g. (Bates and Fife (1993)).

In the infinite domain the critical nucleus is described by a homoclinic trajectory of the equation

$$
w_{x x}=\frac{1}{2 \varepsilon}\left\{\sigma(w)-\sigma\left(w_{0}\right)\right\},
$$

which is readily available in the case of cubic stress-strain relation. The fact that this particular perturbation plays a role of the threshold is illustrated by Fig. 16, clearly indicating sensitivity of dynamics with respect to slight variations of the initial data around this particular profile. In the two numerical experiments presented in Fig. 16, small perturbations $\psi(x)$ and $-\psi(x)$ were added to a profile describing the critical nucleus (homoclinic solution of (6.3.2)). In the first case the perturbation resulted in nucleation and growth of the new phase. In the second case the equivalent perturbation of the opposite sign caused the deterioration of the critical nucleus and the eventual return to the uniform metastable profile.

It is instructive to compare the energy of the critical nucleus with the energies of the critical perturbations of other shapes discussed in Section 6.2 (see also Fig.15). For the critical nucleus we obtain

$$
\Delta E_{c}=\int_{-\infty}^{\infty} f(w)-f\left(w_{0}\right)-\sigma\left(w_{0}\right)\left(w-w_{0}\right)+\varepsilon w_{x}{ }^{2}
$$

which can also be rewritten as 


$$
\frac{\Delta E_{c}}{\sqrt{\varepsilon}}=2 \int_{w_{0}}^{w_{\max }} \sqrt{f(z)-f\left(w_{0}\right)-\sigma\left(w_{0}\right)\left(z-w_{0}\right)} .
$$

We notice that the expression in the right hand side of (6.3.4), measuring the energy density associated with the critical nucleus, is independent of $\varepsilon$. The graph of $\Delta E_{c} / \sqrt{\varepsilon}$ as a function of $w_{0}$ is presented in Fig. 17 (a). As we see inside the metastable domain the size of the barrier diminishes to zero between the Maxwell state $\left(w_{0}=0\right)$ and the spinodal state $\left(w_{0}=0.217\right)$.

Now, consider $\Delta E$, the energy of a critical perturbation from the special classes studied in Section 6.2 (sinusoidal, power law, etc.). According to Fig. 17(b), the threshold $\Delta E_{c}$ corresponding to the critical nucleus (6.3.3) lies in the $\Delta E-w_{0}$ space below every other threshold. This observation supports the idea that $\Delta E_{c}$ represents the lower bound for the activation energy required for triggering the decomposition of a metastable state. We notice that both the energy of the critical nucleus and the size of its support are proportional to $\sqrt{\varepsilon}$. In the limit $\varepsilon \rightarrow 0$, the energy of the critical perturbation goes to zero; however the associated energy density (6.3.4) remains finite depending on $w_{0}$ (or associated applied stress) only. This observation suggests that the nucleation criterion can be formulated in terms of the energy density of the exterior measure-valued "noise". As we have seen in the analysis of the self-similar solutions, this noise is a purely microlevel effect, which is invisible at the continuum level ( see Section 2.5).

\subsection{Non-isothermal nucleation}

The non-isothermal problem encompasses all the complications of the isothermal problem plus the additional ones due to the non-monotonicity of the mobility curves. Indeed, as we have shown previously, in the adiabatic setting the degenerate Riemann problem (2.3.3) may have two non-trivial self-similar solutions, both describing the process of nucleation and growth. The direct dynamic simulation of the nucleation phenomenon in the regularized setting can provide valuable insights concerning stability of the corresponding self-similar solutions and the nature of the attractors for the generic initial data with a localized support.

In this Section, we formally generalize our discrete model from Section 6.1 to the adiabatic case by adding the temperature field $T$ as an additional discrete variable. To include the latent heat effects, we modify the spring response functions $f_{1}$ and $f_{2}$ in the following manner

$$
\begin{gathered}
f_{1}^{\prime}(w, T)=d_{1}+2 a_{1} w+3 b_{1} w^{2}+4 c_{1} w^{3}+B_{1} T \\
f_{2}^{\prime}(w, T)=d_{2}+2 a_{2} w+3 b_{2} w^{2}+4 c_{2} w^{3}+B_{2} T
\end{gathered}
$$

where 


$$
B_{1}=\left.\frac{4}{3} \frac{\partial \sigma}{\partial T}\right|_{w}, B_{2}=-\left.\frac{1}{6} \frac{\partial \sigma}{\partial T}\right|_{w} .
$$

With these modifications, our finite difference-differential equation for the displacement field takes the form

$$
\begin{aligned}
\ddot{u}_{n} & =f_{1}\left(\frac{u_{n+1}-u_{n}}{a}, \frac{T_{n+1}+T_{n}}{2}\right)+f_{2}\left(\frac{u_{n+2}-u_{n}}{2 a}, \frac{T_{n+2}+T_{n}}{2}\right) \\
& -f_{1}\left(\frac{u_{n}-u_{n-1}}{a}, \frac{T_{n}+T_{n-1}}{2}\right)-f_{2}\left(\frac{u_{n}-u_{n-2}}{2 a}, \frac{T_{n}+T_{n-2}}{2}\right) \\
& +\eta\left(\dot{u}_{n+1}-2 \dot{u}_{n}+\dot{u}_{n-1}\right)
\end{aligned}
$$

The corresponding long wave approximation

$$
\frac{\partial^{2} u}{\partial t^{2}}=\left.\frac{\partial \sigma}{\partial w}\right|_{T} \frac{\partial^{2} u}{\partial x^{2}}+\left.\frac{\partial \sigma}{\partial T}\right|_{w} \frac{\partial T}{\partial x}-2 \varepsilon \frac{\partial^{4} u}{\partial x^{4}}+\eta \frac{\partial^{3} u}{\partial t \partial x^{2}}
$$

is equivalent to one of the equations used in the TVC model (3.1.8-9). To identify a discrete equation for "temperature", we first rewrite the continuous entropy equation

$$
\frac{\partial s}{\partial t}=\frac{1}{T}\left\{\kappa \frac{\partial^{2} T}{\partial x^{2}}+\eta\left(\frac{\partial^{2} u}{\partial x \partial t}\right)^{2}\right\}
$$

in the form

$$
\frac{\partial T}{\partial t}=\frac{1}{\partial s / \partial T}\left\{\frac{1}{T}\left(\kappa \frac{\partial^{2} T}{\partial x^{2}}+\eta\left(\frac{\partial^{2} u}{\partial x \partial t}\right)^{2}\right)-\frac{\partial s}{\partial w} \frac{\partial^{2} u}{\partial x \partial t}\right\},
$$

where $s=s(w, T)$. We now discretize (6.4.5) to obtain

$$
\begin{aligned}
\dot{T}_{n} & =\frac{1}{\partial s / \partial T}\left\{\frac { 1 } { T _ { n } } \left[\kappa\left(\frac{4}{3}\left[\frac{T_{n+1}-2 T_{n}+T_{n-1}}{a^{2}}\right]-\frac{1}{12}\left[\frac{T_{n+2}-2 T_{n}+T_{n-2}}{a^{2}}\right]\right)\right.\right. \\
& \left.+\eta\left(\frac{2}{3}\left[\frac{\dot{u}_{n+1}-\dot{u}_{n-1}}{2 a}\right]-\frac{1}{12}\left[\frac{\dot{u}_{n+2}-\dot{u}_{n-2}}{2 a}\right]\right)^{2}\right] \\
& \left.-\frac{\partial s}{\partial w}\left(\frac{2}{3}\left[\frac{\dot{u}_{n+1}-\dot{u}_{n-1}}{2 a}\right]-\frac{1}{12}\left[\frac{\dot{u}_{n+2}-\dot{u}_{n-2}}{2 a}\right]\right)\right\} .
\end{aligned}
$$


One can show that (6.4.5) and (6.4.6) agree with each other within $O\left(a^{4}\right)$.

Now by using the discrete model $(6.4 .2,6.4 .6)$ we can study the non-self-similar stage of the non-isothermal nucleation process in more detail. The approach here will be the same as in the isothermal problem and we present our results as a set of figures analogous to the ones discussed in Section 6.2. Thus, Fig. 18 shows the typical strain and temperature profiles during the dynamical breakdown of the metastable state. In Fig. 19, we compare our dynamic simulations with the semi-analytical self-similar solutions from Section 5. The comparison shows that the discretization is adequate and that our numerical results closely agree with the curves obtained before. We notice, however that the regularized model allows one to reproduce only one of the two self-similar solutions describing nucleation.

Indeed, in the dynamic simulations with $\mathbf{W}_{1}=1, \mathbf{W}_{2}=40$ and $\mathbf{W}_{3}=0.03$, we observe that when the initial strain $w_{0}$ lies between the values 0.083 and 0.123 , the numerical solution approaches only one of the two self-similar regimes, namely the one represented by the lower branch of the nucleation curve (see Fig. 19). We also notice that the stable solutions contain only those kinks which are located on the ascending section of the mobility curve where the driving force grows with the kink velocity.

To explore this issue further, we prescribed special initial data corresponding exactly to point $A$ from Fig. 19 and let the solution evolve according to our regularized model. The numerical experiments show that such a profile immediately breaks down into an alternative system of waves. The initial stage of the associated wave splitting process is detailed in Fig. 20. Similar phenomena were observed when the initial data were taken at the points $B$ and $C$. At the same time, for the points on the lower branch of the nucleation curve, analogous tests show stable evolution, indicating that the corresponding solutions are stable. These numerical observations lend evidence that solutions represented by the upper branch of the nucleation curve are unstable. We emphasize that the corresponding kinks all located on the descending branch of the associated mobility curve (see Fig. 9). Rigorous proof of the instability of these kinks presents an interesting challenge (see related work of Benzoni-Gavage (1999), Corli and Sable-Tougeron (2000) and Zumbrun (2000)).

\section{Conclusions}

The paper addresses the issue of explosive nucleation of a new phase in the framework of the most elementary theory of thermo-elastic rods. In the classical scenario, the decomposition of a metastable state can be simulated by a self-similar solution of a degenerate Riemann problem. However, this Riemann problem, even with kinetic relation specified, is ill-posed and the non-uniqueness occurs among regimes with nucleation and the trivial regime with no new phase forming. This ill-posedness makes it necessary to abandon the self-similar setting and return to the solution of the more complex, micro-level problem with regularized initial data. With the goal of finding the limits of stability associated with a given metastable state we studied evolution of the 
localized perturbations superimposed on this metastable state. Our main result is a quantitative connection between the possibility of nucleation and the size of the domain of attraction of the trivial regime describing metastable equilibrium.

We interpret the non-uniqueness in the original thermo-elastic problem as an indicator of the essential interaction between continuum and sub-continuum scales. It is important to remember that the classical continuum theory represents a long wave approximation to the behavior of a structured medium (atomic lattice, layered composite, plate of finite thickness) and as such does not contain information about the processes at small scales which are effectively homogenized out. In some cases the detailed behavior at the micro-level turns out to be irrelevant and the closure can be achieved by prescribing some very general thermodynamic constraints like the entropy inequality. This situation can be illustrated by the fact that in the hyperbolic systems the fine structure of the shock discontinuity does not affect the dynamics and that singular perturbations in the initial data die out instantly. If the energy at the micro-level is nonconvex, like in the case of phase transitions, the situation is more complex and in order to obtain a unique solution at the continuum level, one must "de-homogenize" the naïve macro model and introduce additional physical hypotheses about the behavior at the subcontinuum scales. This kind of regularization is achieved automatically in numerical calculations because of the dissipation and dispersion which discretization itself brings into the model.

In this paper, we use the proven TVC model as a prototypical micro-description and show how the information about the behavior of the solutions at the micro-scale can be used to narrow the non-uniqueness at the macro-scale. The gradient part of this model contains a small parameter with a scale of length $(\sqrt{\varepsilon})$, and with other small parameters scaled accordingly the classical thermo-elasticity can be viewed as a limit of this "micromodel" as this parameter tends to zero. Through the study of the nucleation phenomenon we have shown that the localized perturbations of the form $\phi(x / \sqrt{\varepsilon})$ can influence the choice of the attractor for the limiting problem. We observe that for this type of perturbations, support but not amplitude vanishes as the small parameter $\sqrt{\varepsilon}$ goes to zero. The same phenomenon is the dependence of the limiting solution on the contributions of the type $\varphi((x-D t) / \sqrt{\varepsilon})$, describing the structure of the kink and generating specific kinetic relations. As we see in this problem not only the limit but also the character of convergence matters. This suggests that consistent limit of the TVC regularization of the continuum theory with non-convex energy should be formulated in a broader functional space than is currently accepted in the classical thermo-elasticity. That is why, our nucleation criterion detailed in Fig. 15, 17 is presented in terms of intensity of the perturbations, a parameter which remain invisible in the classical setting.

\section{Acknowledgments}

This work was supported by the NSF grant DMS-9803572. The authors would like to thank Bernardo Cockburn for many valuable discussions. 


\section{References}

[AK91a] Abeyaratne R., Knowles J.K., Kinetic relations and the propagation of phase boundaries in solids, Arch. Rat. Mech. Anal. 114, 119-154 (1991).

[AK91b] Abeyaratne R., Knowles J.K., Implications of viscosity and strain-gradient effects for the kinetics of propagating phase boundaries in solids, SIAM J. Appl. Math. 51: 1205-1221 (1991).

[AK94] Abeyaratne R., Knowles J.K., Dynamics of propagating phase boundaries: thermoelastic solids with heat conduction., Arch. Rat. Mech. Anal. 126, 203-230 (1994).

[ACJ96] Abeyaratne R., Chu C., James, R.D., Kinetics of materials with wiggly energies: theory and application to the evolution of twinning microstructures in a Cu-Al-Ni shape memory alloy. Phil. Mag. A 73: 457-497 (1996).

[BCS01a] Balk, A.M., Cherkaev, A.V., Slepyan, L.I., Dynamics of chains with non-monotone stress-strain relations. I. Model and numerical experiments, J. Mech. Phys. Solids 49: 131-148 (2001).

[BCS01b] Balk, A.M., Cherkaev, A.V., Slepyan, L.I., 2000b. Dynamics of chains with non-monotone stress-strain relations. II. Nonlinear waves and waves of phase transition, J. Mech. Phys. Solids 49: 149-171 (2001).

[BF93] Bates P.W., Fife P.C. The dynamics of nucleation for the Cahn-Hilliard equation, SIAM J. Appl. Math. 53, 990-1008 (1993).

[B99] Benzoni-Gavage S., Stability of subsonic planar phase boundaries in a van der Waals fluid. Arch. Rat. Mech. Anal. 150: 23-55 (1999).

[CG96] Cockburn B., Gao H., A model numerical scheme for the propagation of phase transitions in solids. SIAM J. Sci. Compt. 17: 1092-1121 (1996).

[CL01] Chalons C., LeFloch P.G., Higher order entropy conservative schemes and kinetic relations for van der Waals fluids. J. Comp. Phys. (2001).

[CS00] Corli A., Sable-Tougeron M., Kinetic stabilization of a nonlinear sonic phase boundary. Arch. Rat. Mech. Anal. 152: 1-63 (2000).

[CT00] Charlotte M., Truskinovsky L., Linear chains with a hyper-pre-stress, J. Mech. Phys. Solids, (2001)

[D73] Dafermos C.M., The entropy rate admissibility criterion for solutions of hyperbolic conservation laws, J. Diff. Eq. 14: 202-212 (1973).

[E75] Ericksen J.L., Equilibrium of bars, J. Elasticity 5: 191-202, (1975).

[FL95] Frid H., Liu I-S., Oscillation waves in Riemann problems inside elliptic regions for conservation laws of mixed type, ZAMP 46: 913-931 (1995).

[G93] Gurtin M.E., The dynamics of solid phase transitions 1. Coherent interfaces, Arch. Rat. Mech. Anal. 123: 305-335 (1993).

[J80] James, R.D., The propagation of phase boundaries in elastic bars, Arch. Rat. Mech. Anal. 73: 125-158 (1980).

[HHL76] Harten A., Hyman J.M., Lax P.D. On finite difference approximations and entropy conditions for shocks, Comm. Pure Appl. Math. 29:297-322(1976)

[K82] Kunin I.A., Elastic Media with Microstructure I: One Dimensional Models, Springer Series in Solid States Science, v. 26 (1982).

[L71] Lax P.D., Shock waves and entropy, In: Contributions to Nonlinear Functional Analysis. E.A. Zarantonello, Ed., Academic Press, NY (1971).

[LP98] Lin J., Pence T.J., Pulse attenuation by kinetically active phase boundary scattering during displacive phase transformations. J. Mech. Phys. Solids 46: 1183-1211 (1998).

[LR00] LeFloch P.G., Rohde C., High-order schemes, entropy inequalities, and nonclassical shocks, SIAM J. Numer. Anal. 37: 2023-2060 (2000).

[LSB93] Leo P.H., Shield T.W., Bruno, O.P., Transient heat transfer effects on the pseudoelastic behavior of shape-memory wires. Acta Metall. Mater. 41: 2477-2485 (1993).

[MS92] Mihailescu-Suliciu M., Suliciu I., On the method of characteristics in rate type viscoelasticity with non-monotone equilibrium curve, ZAMM 72,667-674 (1992).

[M65] Mindlin R., Second gradient of strain and surface tension in linear elasticity, Int. J. Solids Structures 1: 417-438 (1965).

[NT99] Ngan S.-C., Truskinovsky L., Thermal trapping and kinetics of martensitic phase boundaries. $J$. Mech. Phys. Solids 47: 141-172 (1999).

[OW98] Otsuka K., Wayman C.M (Ed.) Shape Memory Materials. Cambridge University Press (1998). 
[PT01] Puglisi G., Truskinovsky L., Rate-independent hysteresis in a bi-stable chain , J. Mech. Phys. Solids, (2001)

[RT00] Ren X., Truskinovsky L., Finite scale microstructures in nonlocal elasticity, 59:319-355 J. Elast., (2001)

[RH98] Rybka P., Hoffmann, K.H., Convergence of solutions to the equation of quasi-static approximation of viscoelasticity with capillarity. J. Math. Anal. Appl. 226: 61-81 (1998).

[RK97] Rosakis P., Knowles J.K., Unstable kinetic relations and the dynamics of solid-solid phase transitions. J. Mech. Phys. Solids 45:2055-2081 (1997).

[Sh82] Shearer M., The Riemann Problem for a Class of Conservation Laws of Mixed Type, J. Diff. Eq. 46: 426-443 (1982).

[Sh83] Shearer M., Admissibility criteria for shock wave solutions of a system of conservation laws of mixed type, Proc. Royal Soc. Edinburgh, 93: 233-244 (1983).

[Sh86] Shearer M., Nonuniqueness of admissible solutions of Riemann initial value problems for a system of conservation laws of mixed type. Arch. Rat. Mech. Anal. 93: 45-59 (1986).

[SY95] Shearer M., Yang Y., The Riemann problem for a system of conservation laws of mixed type with a cubic nonlinearity, Proc.Roy. Soc. Edinburgh A 125: 675-700 (1995).

[S183] Slemrod M., Admissibility criteria for propagating phase boundaries in a Van der Waals fluid, Arch. Rat. Mech. Anal. 81: 301-315 (1983).

[S184a] Slemrod M., Dynamic phase transitions in a van der Waals fluid, J. Diff. Eqs. 52: 1-23, (1984).

[S184b] Slemrod M., Lax-Fredricks and the viscosity-capillarity criterion. In: Physical Partial Differential Equations, J. Lightbourne, S. Rankin (Eds), Marcel Dekker, NY, 75-84 (1984).

[SF86] Slemrod M., Flaherty J.E., Numerical investigation of a Riemann problem for a van der Waals fluid, in: Phase Transformations, E.C.Aifantis, J.Gittus (Eds.), Elsevier, 203-212 (1986).

[SLG97] Shield, T.W.P., Leo H., Grebner, W.C.C., Quasi-static extension of shape memory wires under constant load. Acta Mater. 45: 67-74 (1997).

[S00] Slepyan, L.I., Dynamic factor in impact, phase transition and fracture. J. Mech. Phys. Sol. 48: 927960 (2000).

[S01] Slepyan, L.I., Feeding and dissipative waves in fracture and phase transition. II. Phase transition waves. J. Mech. Phys. Sol. 49: 513-550 (2001).

[ST84] Slepyan, L.I., Troiankina, L.V., Fracture wave in a chain structure. J. Appl. Mech. Tech. Phys. 25, 921-927 (1984).

[T82] Truskinovsky L., Equilibrium phase boundaries, Sov. Phys. Doklady 27: 551-553 (1982).

[T85] Truskinovsky L., Structure of an isothermal phase jump, Sov. Phys. Doklady 30: 945-948 (1985).

[T87] Truskinovsky L., Dynamics of nonequilibrium phase boundaries in a heat conducting nonlinear elastic medium, J.Appl. Math. and Mech. (PMM) 51: 777-784 (1987).

[T93a] Truskinovsky L., About the normal growth approximation in the dynamical theory of phase transitions, Cont. Mech. and Therm. 6: 185-208 (1993).

[T93b] Truskinovsky L., Kinks versus Shocks. In: Shock Induced Transitions and Phase Structures in General Media, R. Fosdick, E. Dunn and M. Slemrod (Eds.), IMA 52, Springer-Verlag, (1993).

[T94] Truskinovsky L., Transition to detonation in the dynamic phase changes, Arch. Rat. Mech. Anal. 125: 375-397 (1994).

[T97] Truskinovsky L., Nucleation and growth in elasticity. In: Dynamics of Crystal Surfaces and Interfaces, P. Duxbury and T. Pence (Eds.), Plenum Press, New York (1997)

[Tu97a] Turteltaub S., Viscosity and strain gradient effects on the kinetics of propagating phase boundary in solids, J. Elast. 46: 55-90 (1997).

[Tu97b] Turteltaub S., Adiabatic phase boundary propagation in a thermoelastic solid. Math. Mech. Solids 2:117-142 (1997).

[TB93] Triantafyllidis N., Bardenhagen S., On higher order gradient continuum theories in 1-D nonlinear elasticity. Derivation from and comparison to the corresponding discrete models. J. Elast. 33: 259-293 (1993).

[V99] Vainchtein A., Dynamics of phase transitions and hysteresis in a viscoelastic Ericksen's bar on an elastic foundation. J. Elast. 57: 243-280 (1999).

[V01] Vainchtein A., Stick-slip motion of interfaces as a singular limit of the viscosity-capillarity model. Mathematics and Mechanics of Solids, 6:323-342 (2001).

[VR99] Vainchtein A., Rosakis P., Hysteresis and stick-slip motion of phase boundaries in dynamic models of phase transitions, Journal of Nonlinear Science 9: 697--719 (1999). 
[Z00] Zumbrun K., Dynamical stability of phase transitions in the p-system with viscosity-capillarity. SIAM J. Applied Math. 60: 1913-1924 (2000). 


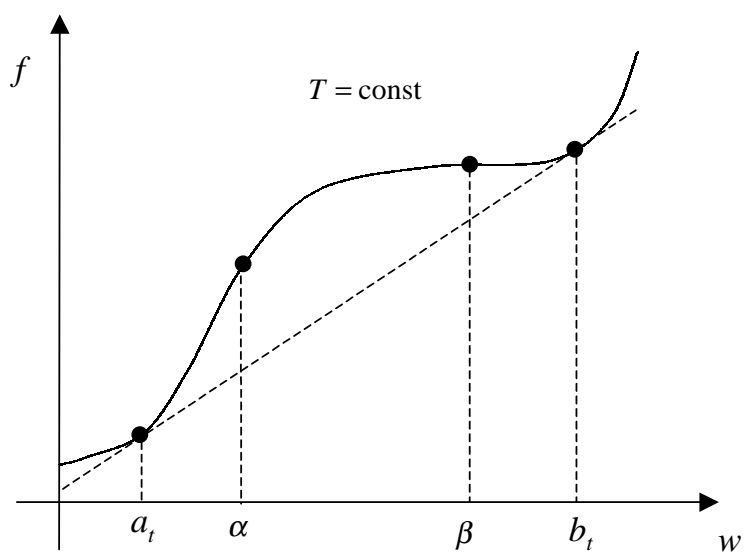

(a)

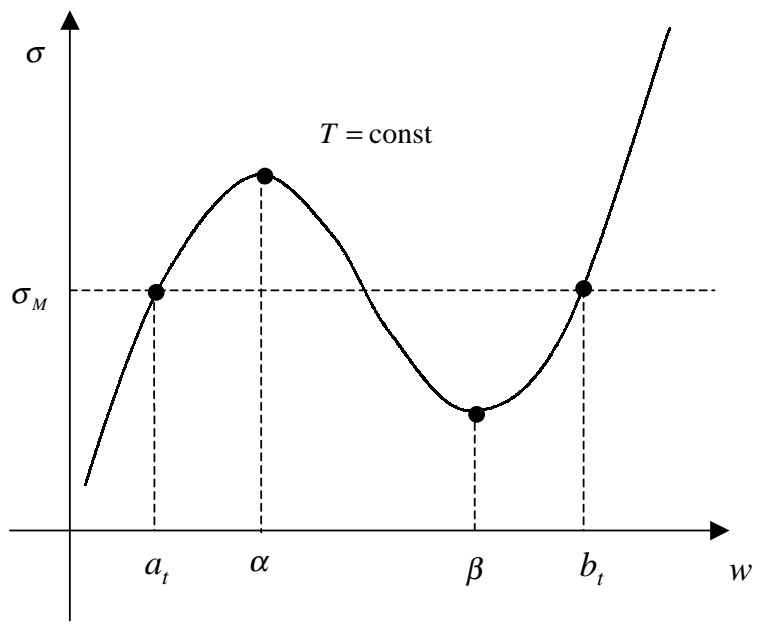

(b)

Fig. 1. The non-convex free energy density at constant temperature (a) and the associated non-monotone stress-strain relation (b) for an elastic material supporting two phases.

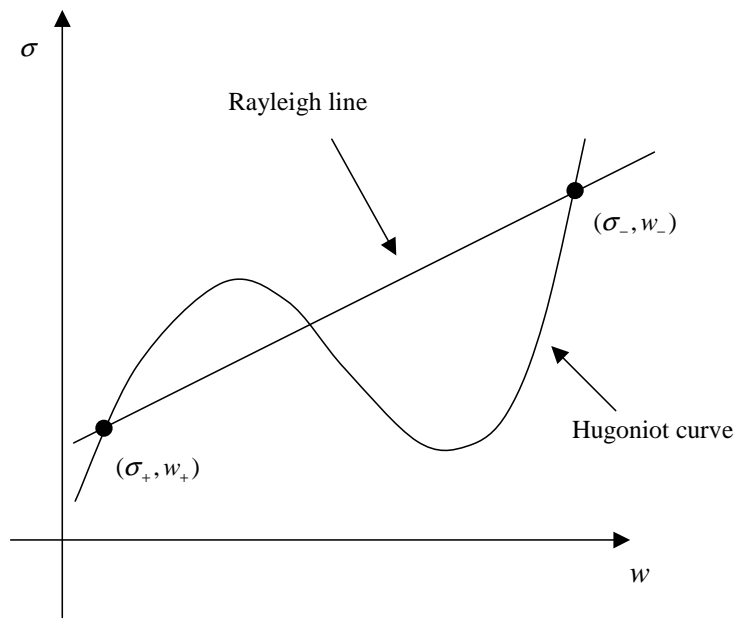

Fig. 2. Relative position of the Hugoniot adiabat and the Rayleigh line for an elastic material supporting two phases. 


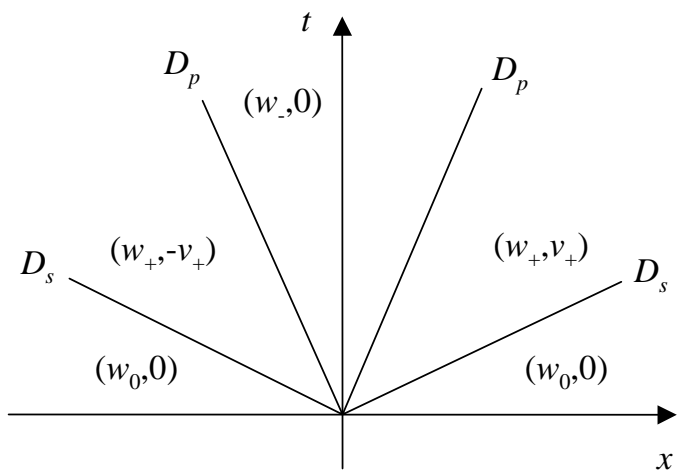

(a)

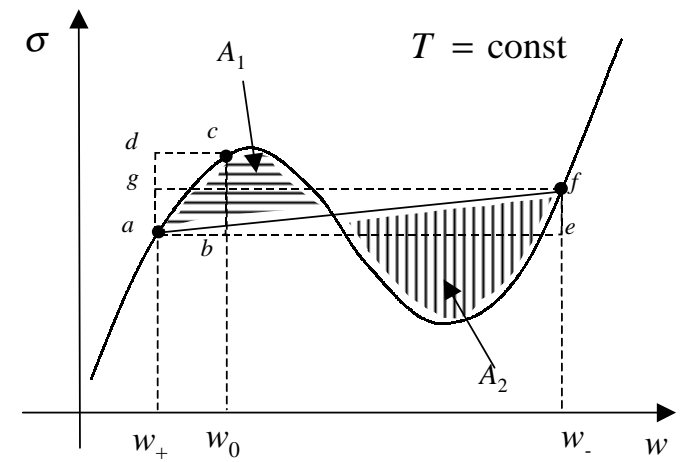

(b)

Fig.3. The self-similar solution describing isothermal nucleation of a new phase with the emission of two shock wave precursors: trajectories of the discontinuities (a); relation between stresses and strains ahead and behind the discontinuities (b).

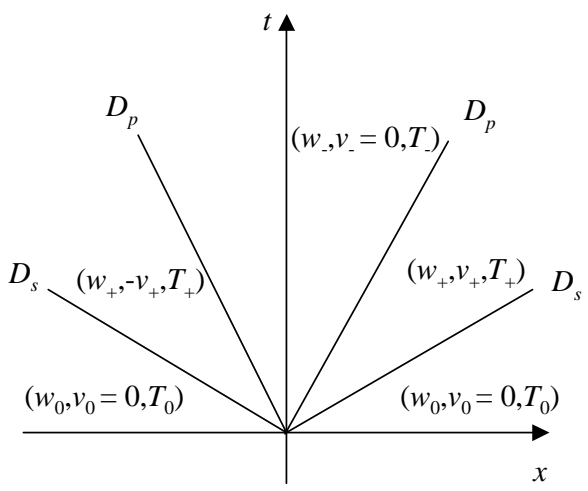

(a)

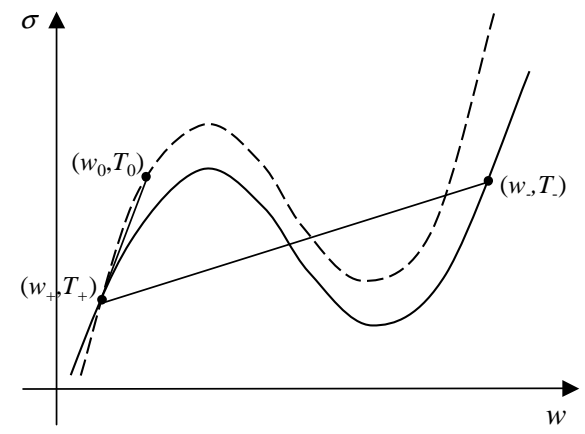

(b)

Fig. 4 The self-similar solution describing nucleation in the adiabatic case: trajectories of the discontinuities (a); relation between stresses and strains ahead and behind the discontinuities (b). 


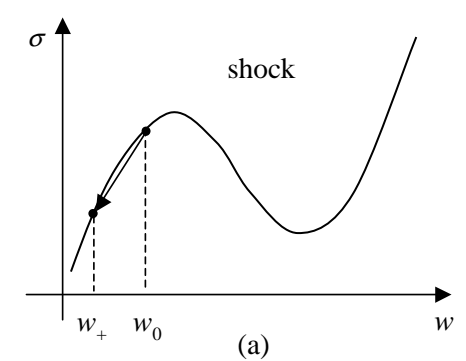

(a)

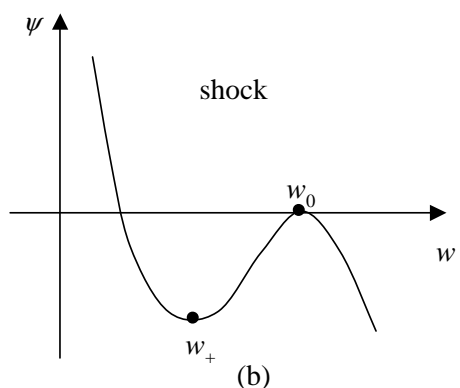

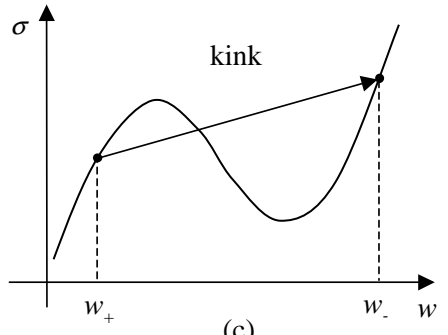

(c)

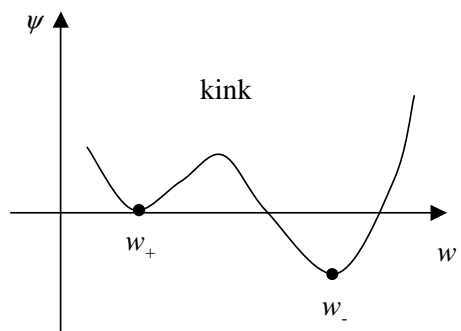

(d)

Fig.5. Illustration of the difference between the fine structure of the energy release inside shocks $(a, b)$ and inside kinks (c,d). See the main body of the paper for more details.

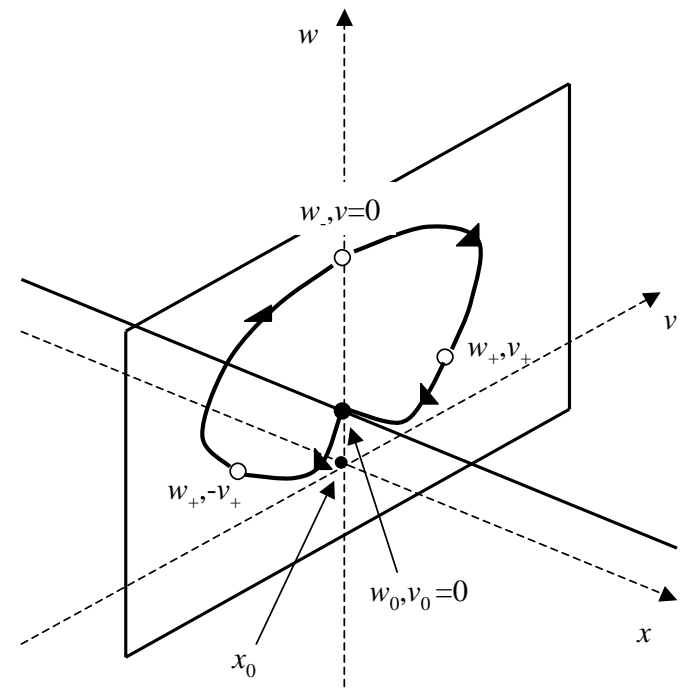

Fig.6. A finite loop in the $(w, v)$ space superimposed on the piecewise constant initial data constituting the degenerate Riemann problem. 


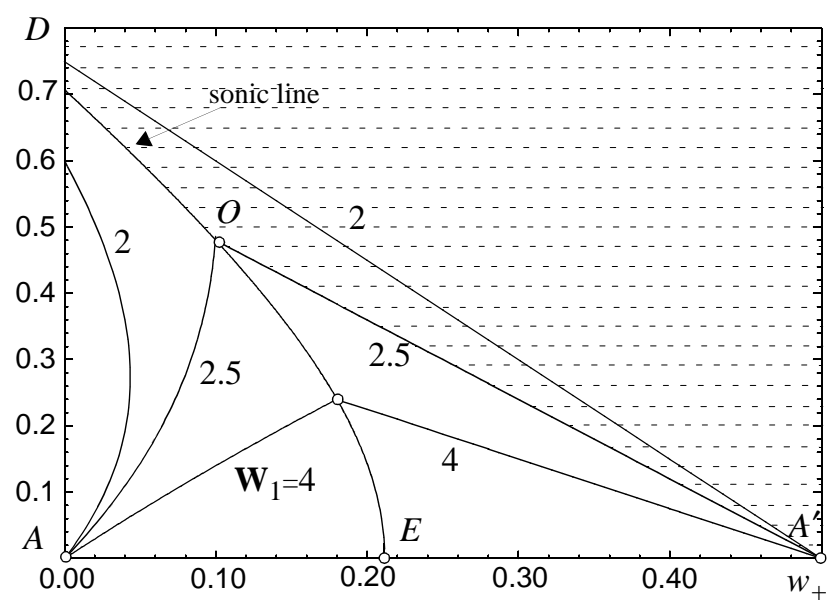

Fig. 7. Kinetic curves for the isothermal kinks at various $\mathbf{W}_{1}$. The shaded domain contains admissible shocks for $\mathbf{W}_{1}=2.5$.

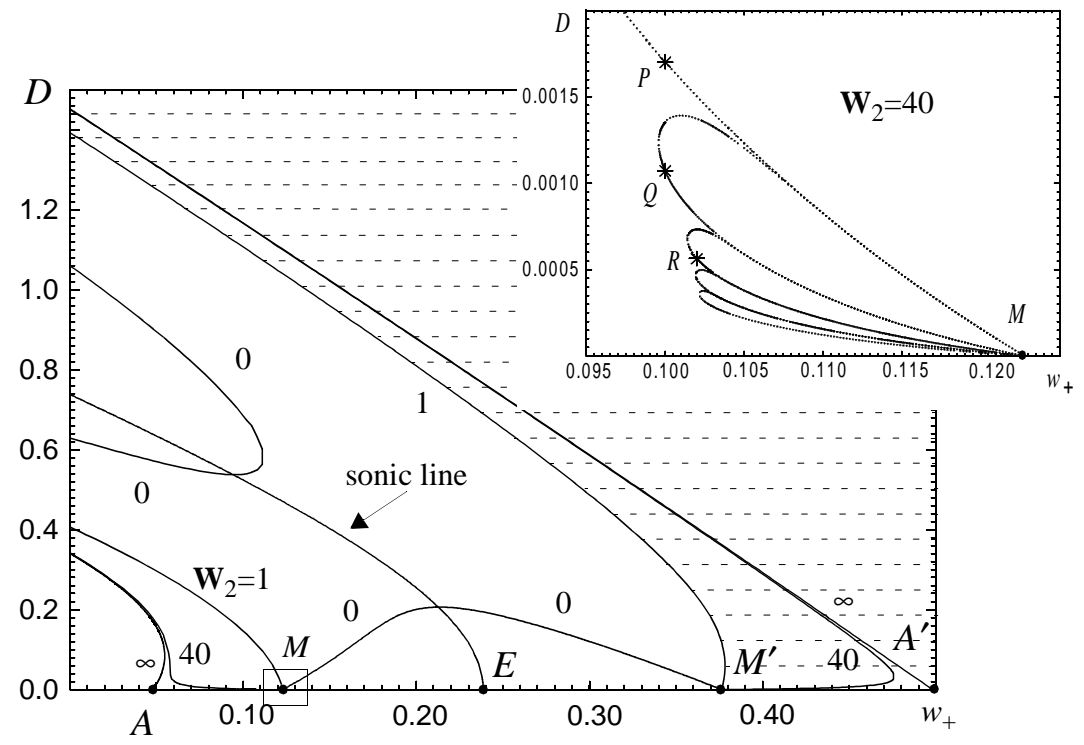

Fig. 8 Kinetic curves for the general non-isothermal problem at different values of $\mathbf{W}_{2}$ with $\mathbf{W}_{1}=1$ and $\mathbf{W}_{3}$ $=0.03$. The upper right inset shows the blow up of the loop structure near the point $M$ for $\mathbf{W}_{2}=40$. The shaded region corresponds to admissible shocks at $\mathbf{W}_{2}=1$. 


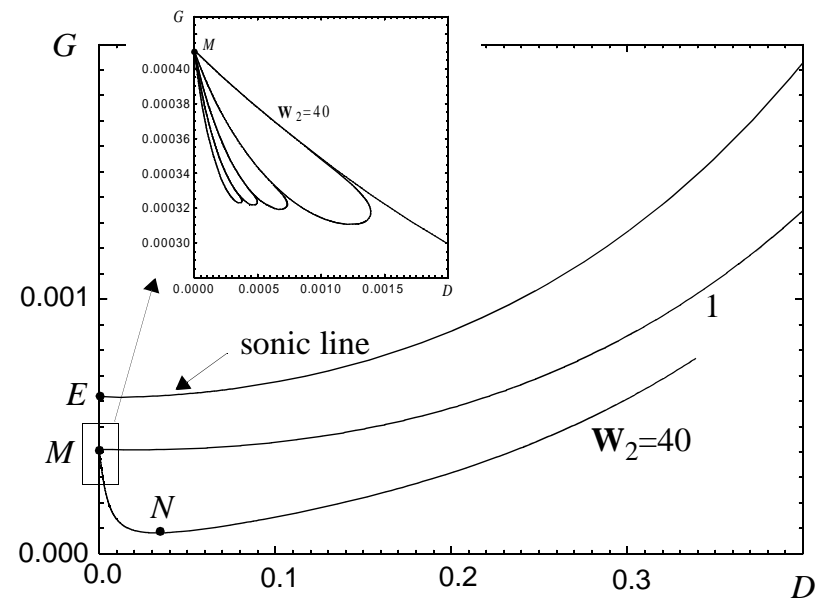

Fig. 9 General structure of the mobility curves for the TVC model at different values of $\mathbf{W}_{2}$ with $\mathbf{W}_{1}=1$ and $\mathbf{W}_{3}=0.03$. 

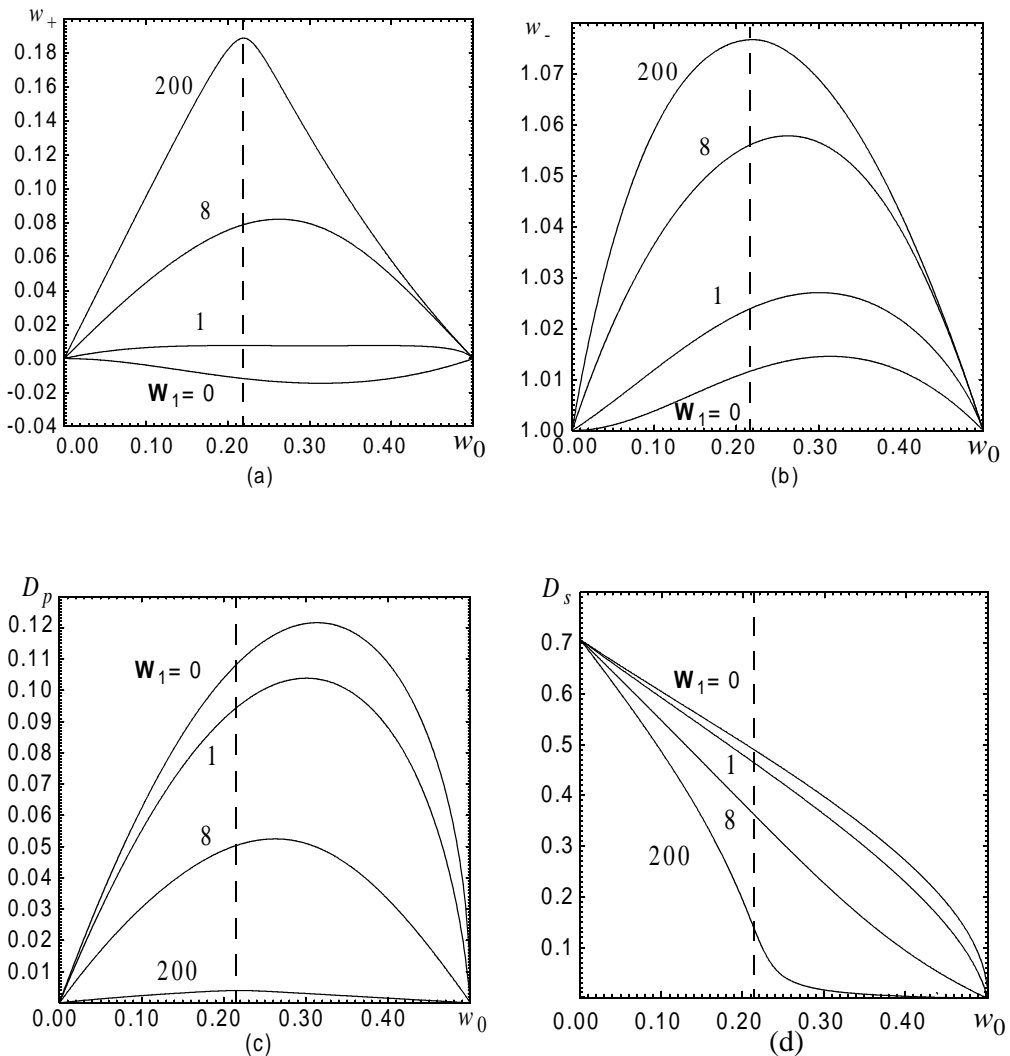

Fig. 10. Parameters of the self-similar solution (2.3.6) describing isothermal decomposition of a metastable state with $\mathbf{W}_{1}$ varying from 0 to 200 . To the right of the dashed line the initial state is unstable (spinodal region), and alternative modes of decomposition are also available. 

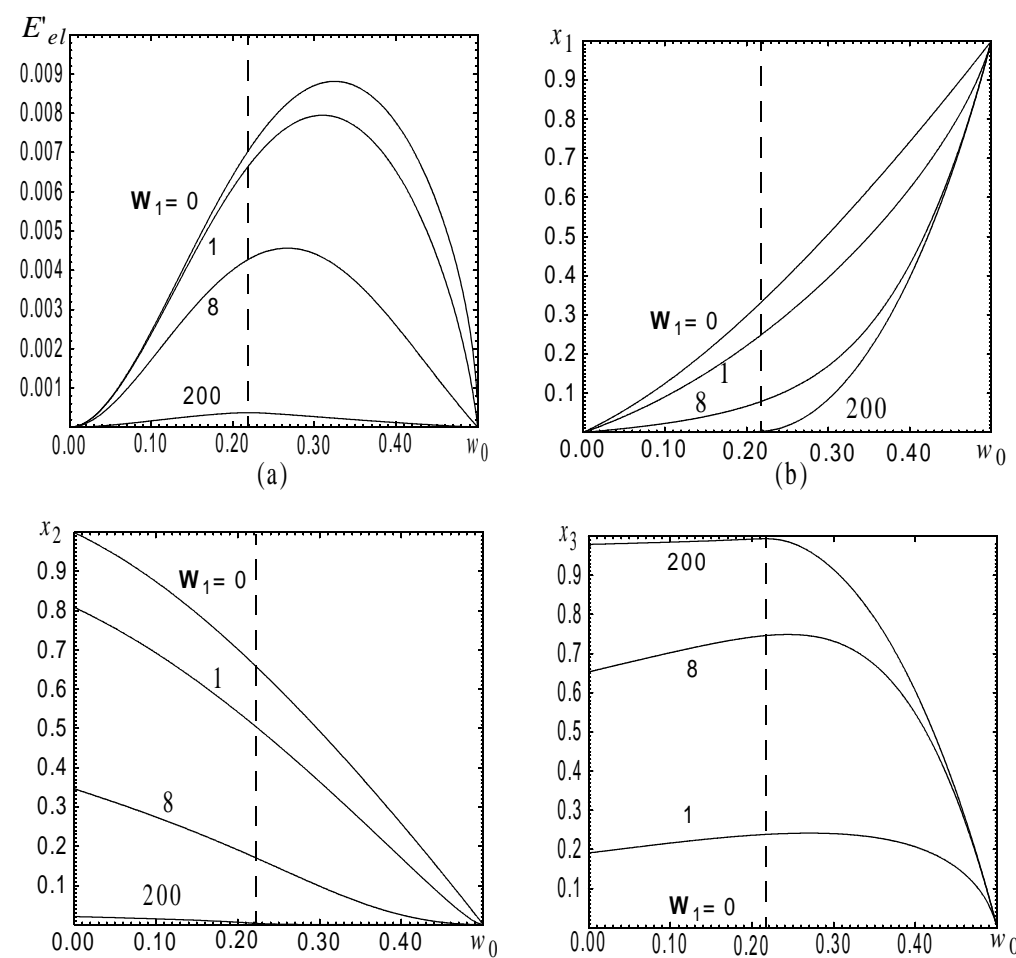

(c)

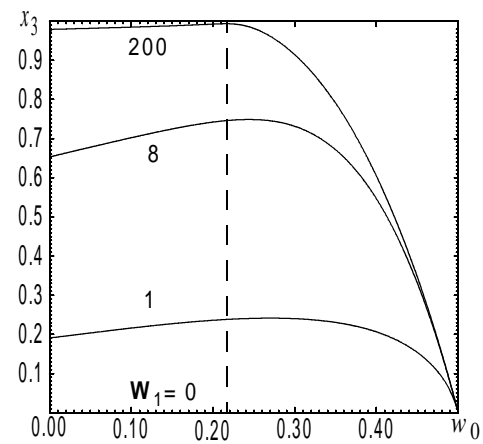

(d)

Fig. 11. Energy redistribution in the isothermal nucleation process (see the text). Parameter $\mathbf{W}_{1}$ varies from 0 to 200 . The curve for $\mathbf{W}_{1}=0$ in (d) lies precisely on the horizontal axis. 


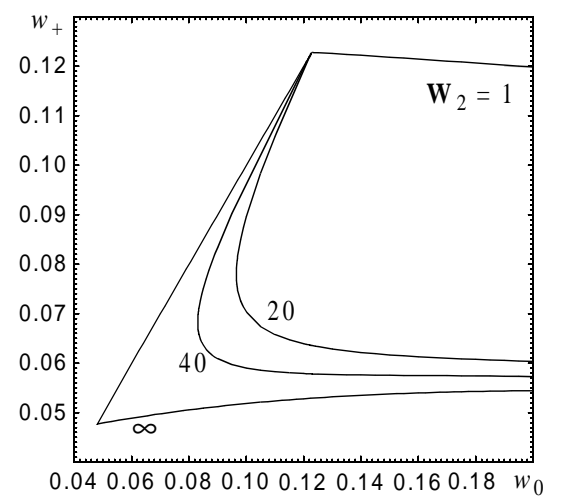

(a)

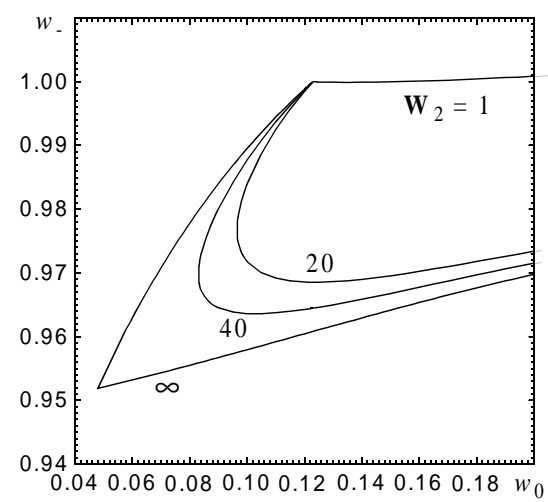

(c)

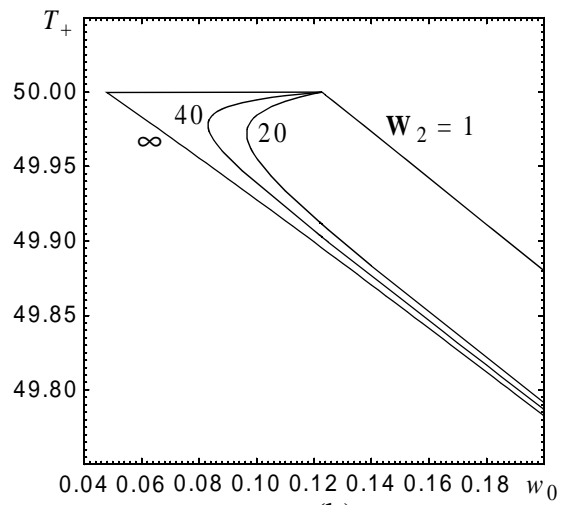

(b)

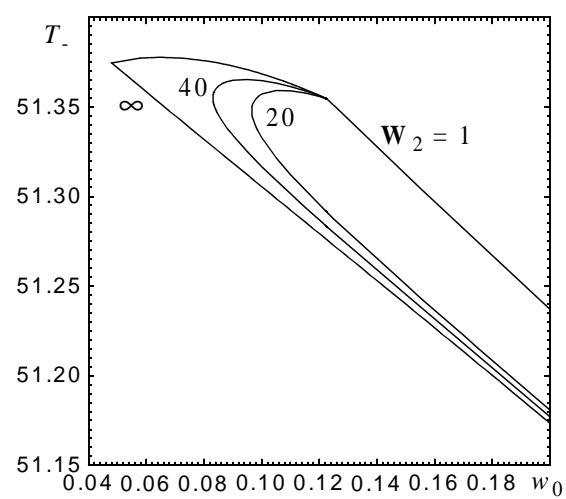

(d)

Fig.12. Parameters of the adiabatic decomposition of a metastable state (2.3.7). Parameter $\mathbf{W}_{2}$ varies from 1 to infinity. $\mathbf{W}_{1}=1, \mathbf{W}_{3}=0.03$ and $T_{0}=50$. 


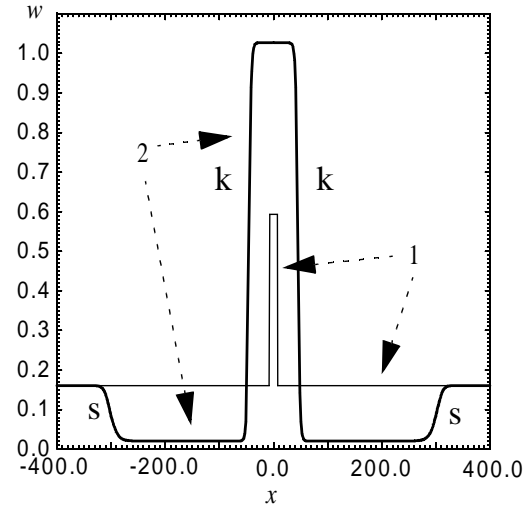

(a)

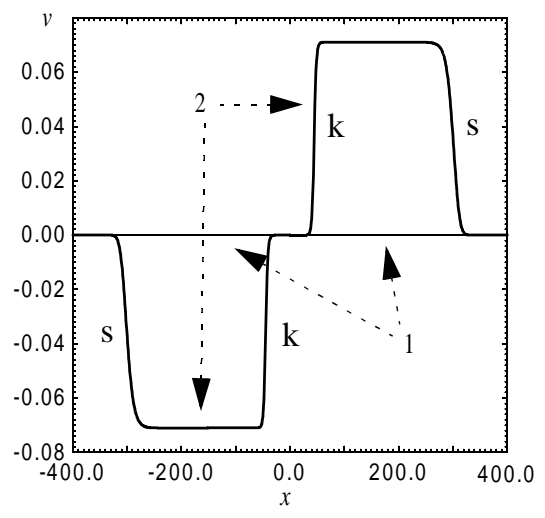

(b)

Fig.13. Strain and velocity profiles in the process of nucleation from a metastable state. Here 1 represents initial perturbation, and 2 the configuration at a later time. Letters $\mathrm{s}$ and $\mathrm{k}$ indicate shocks and kinks.

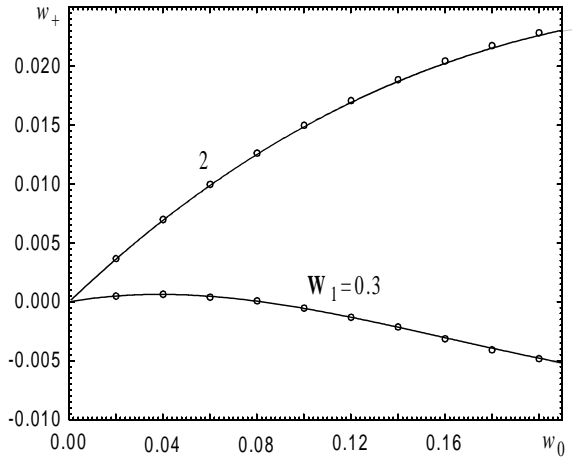

(a)

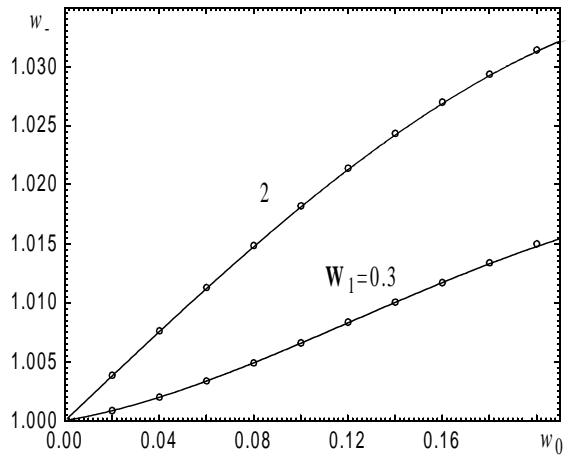

(b)

Fig. 14. Comparison of the isothermal nucleation in the discrete and continuum models. Circles represent the results of numerical simulation in the discrete model, solid lines show semi-analytical results for the continuum model (self-similar solution). 


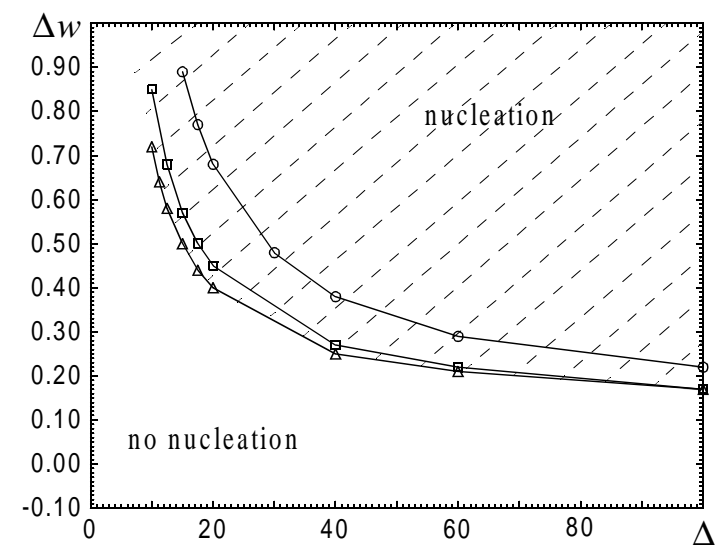

Fig. 15. Minimum strength of the perturbation required to nucleate a new phase from an initially uniform metastable state. Here $\Delta$ corresponds to the width of the perturbation. $\Delta w$ is the maximum amplitude of the perturbation. Triangles represent sinusoidal perturbations, squares - power law perturbations with $n=2$, and circles - power law perturbations with $n=6$. In all cases $w_{0}=0.10$ and $\mathbf{W}_{1}=3$.

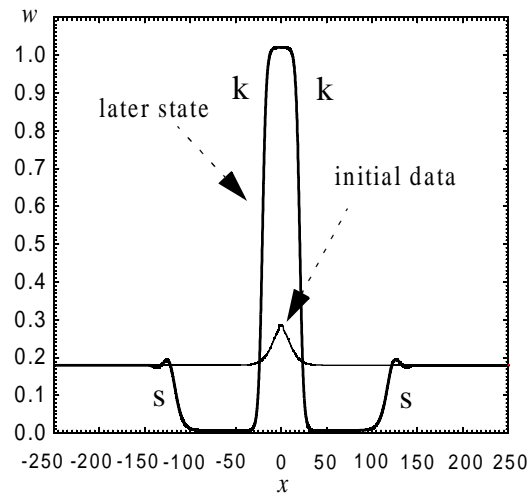

(a)

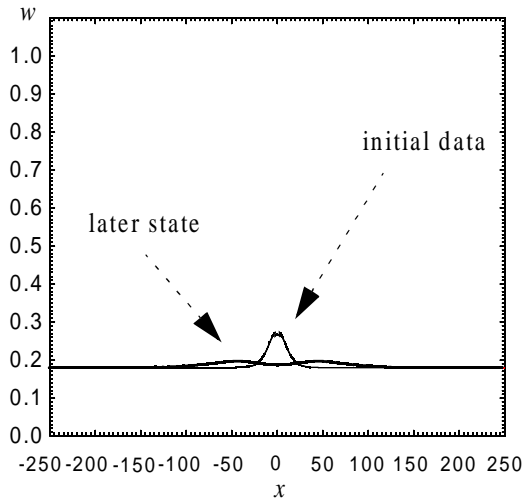

(b)

Fig. 16. Sensitivity of the system to small perturbations superimposed on the configuration corresponding to the critical nucleus. In (a) the critical nucleus is subjected to a small positive perturbation. In (b), the perturbation is of the opposite sign. Two different dynamic regimes emerge: nucleation in case (a) and relaxation in case (b). Letters $\mathrm{s}$ and $\mathrm{k}$ indicate shocks and kinks. 


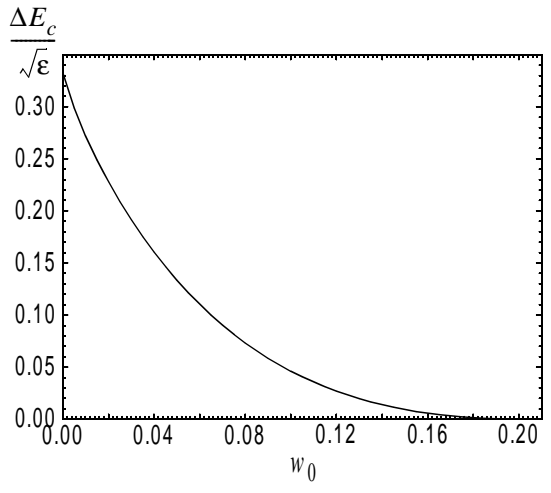

(a)

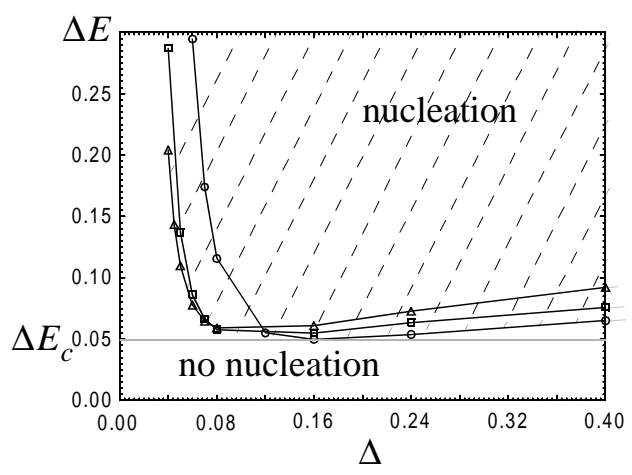

(b)

Fig. 17. The energy of a critical perturbation required to trigger the decomposition of a metastable state: (a) minimal energy (critical nucleus); in (b) triangles represent sinusoidal perturbations, squares - power law perturbations with $n=2$, and circles - power law perturbations with $n=6$.

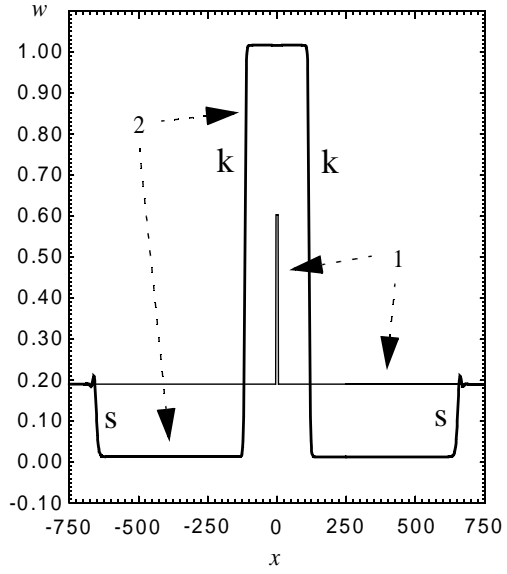

(a)

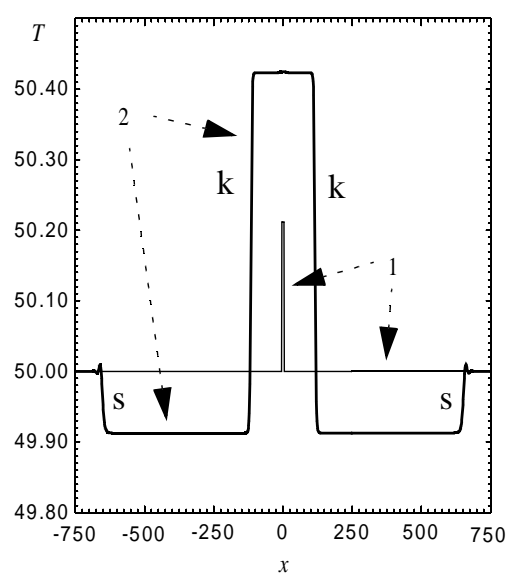

(b)

Fig.18. Strain and temperature profiles during the adiabatic nucleation: 1 - initial perturbation, 2 configuration at a later time. Letters $\mathrm{s}$ and $\mathrm{k}$ indicate shocks and kinks. 

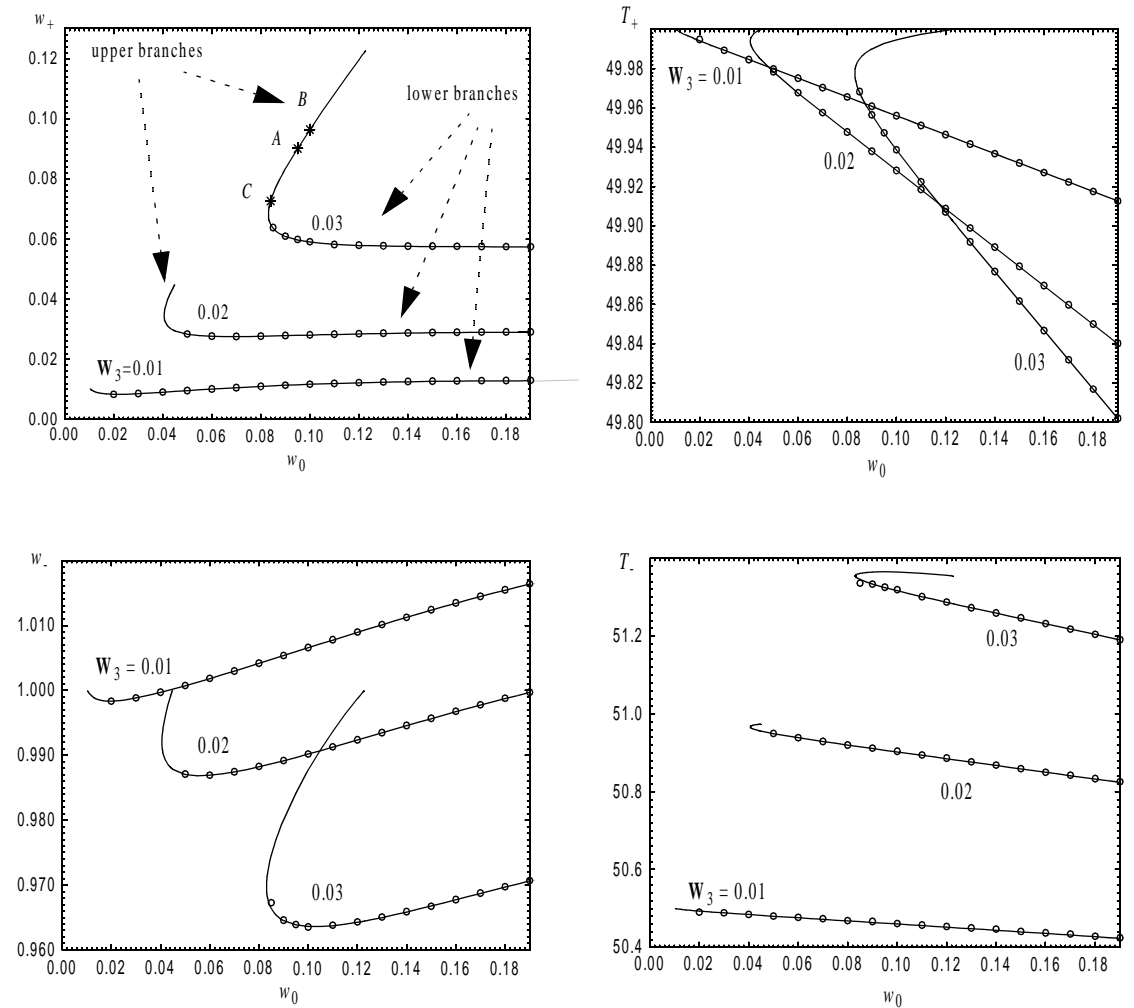

Fig.19. Nucleation in the discrete model compared with the self-similar solutions. Circles represent results of numerical simulation; solid lines correspond to semi-analytical solution from Section 5. Here $\mathbf{W}_{1}=1$ and $\mathbf{W}_{2}=40$. 

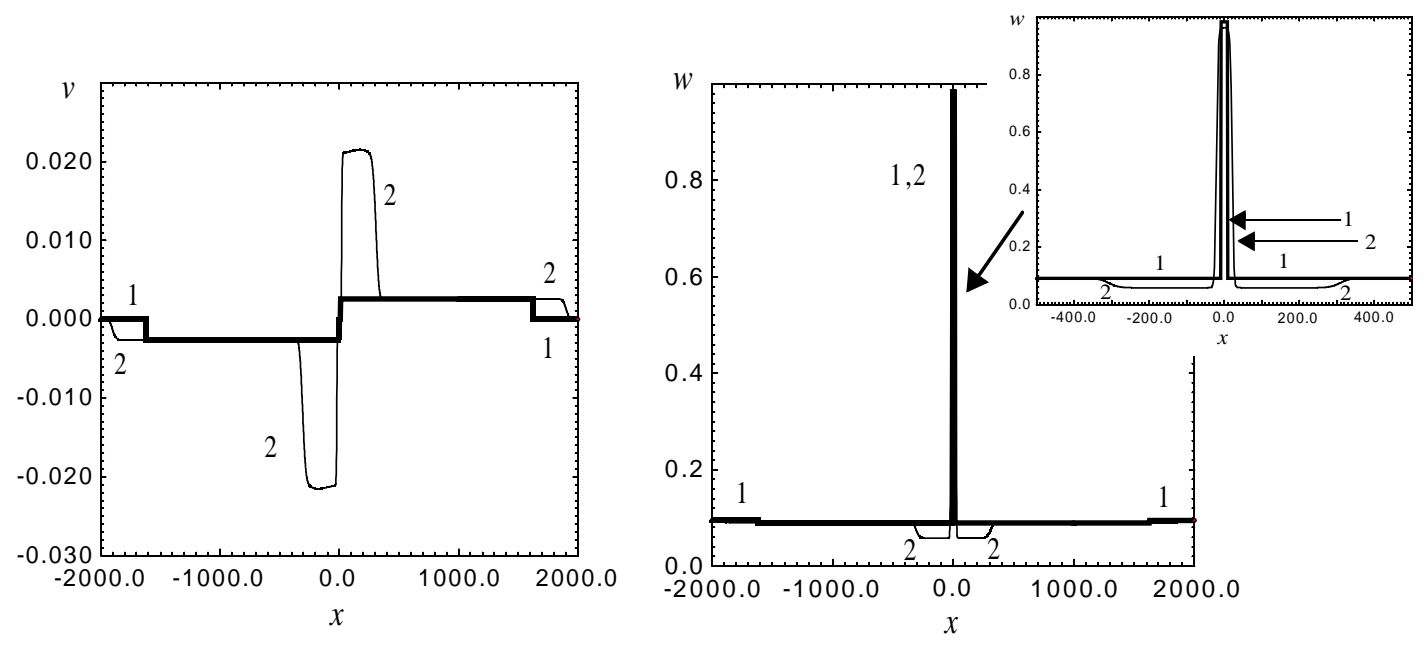

Fig. 20. Spontaneous breakdown of the strain and velocity profiles corresponding to point A in Fig. 19: 1 initial profile corresponding to point A, 2 - subsequent evolution of this profile. The inset shows a magnification of the middle part of the picture. 